\title{
Studies of dense molecular cores in regions of massive star formation
}

\section{Core properties on the galactic scale ${ }^{\star}$}

\author{
I. Zinchenko ${ }^{1,2}$, L. Pirogov ${ }^{1}$, and M. Toriseva ${ }^{2}$ \\ ${ }^{1}$ Institute of Applied Physics of the Russian Academy of Sciences, 46 Uljanov str., 603600 Nizhny Novgorod, Russia \\ ${ }^{2}$ Helsinki University Observatory, Tähtitorninmäki, P.O. Box 14, FIN-00014 University of Helsinki, Finland
}

Received March 25; accepted June 18, 1998

\begin{abstract}
We surveyed 55 northern non-stellar $\mathrm{H}_{2} \mathrm{O}$ masers in the CS $J=2-1$ line with the 20 -m Onsala radio telescope and detected 47 CS cores associated probably with 50 masers. The CS emission for this sample is weaker on the average than for the similar southern sample studied by us earlier at SEST. Most of the detected cores were mapped in CS. The CS peaks were observed also in the $\mathrm{C}^{34} \mathrm{~S} J=2-1$ and in the CO $J=1-0$ lines. We present CS maps as well as CO, CS and, when available, $\mathrm{C}^{34} \mathrm{~S}$ spectra for the 26 best studied cores.

From the CS maps and optically thin $\mathrm{C}^{34} \mathrm{~S}$ emission we derive the basic physical parameters of the cores: size, LTE mass, mean density, virial mass. Combining the present results with the previous SEST data we obtain statistical distributions of the core parameters. The CO brightness temperature distribution for most cores ranges from $\sim 15 \mathrm{~K}$ to $\sim 50 \mathrm{~K}$ with a peak at $20-30 \mathrm{~K}$. The typical sizes of the cores are $L \sim 1.0-1.5$ pc. The mean density lies in the range $n \sim 10^{3}-10^{5} \mathrm{~cm}^{-3}$ which is much lower than densities needed for CS excitation from multitransitional analysis. The slope of the mass spectrum for $M \gtrsim 1000 M_{\odot}$ is $1.6 \pm 0.3$. The ratio of the IR luminosity of associated IRAS point sources to mass peaks at $\sim 10 L_{\odot} / M_{\odot}$. The CS line widths are highly supersonic $\left(\sim 1.5-9 \mathrm{~km} \mathrm{~s}^{-1}\right)$.

We analyze the dependences of these parameters on galactocentric distance $R$. The mean density of the cores drops with increasing $R$ in the interval $R \approx 7-14 \mathrm{kpc}$. It is consistent with an exponential law with a scale length of $\sim 3 \mathrm{kpc}$. The IR luminosity to mass ratio changes probably in a similar way. The core size increases with $R$ in accordance with the density decrease and constant mass.
\end{abstract}

Send offprint requests to: I. Zinchenko

Correspondence to: zin@appl.sci-nnov.ru

* Table 2 is only available in electronic form at the CDS via anonymous ftp to cdsarc.u-strasbg.fr (130.79.128.5) or via http://cdsweb.u-strasbg.fr/Abstract.html
The comparison of the $\mathrm{CS}$ and $\mathrm{C}^{34} \mathrm{~S}$ data shows almost no broadening of the CS lines due to optical depth effects. This can be probably explained by small scale clumpiness in the cores. The velocity difference between the CS cores and $\mathrm{H}_{2} \mathrm{O}$ masers is close to zero on the average with the standard deviation of $\sim 7 \mathrm{~km} \mathrm{~s}^{-1}$.

Key words: stars: formation - ISM: clouds - ISM: molecules — radio lines: interstellar

\section{Introduction}

Statistical properties of dense molecular cores in regions of high mass star formation (HMSF) and their possible variations on the galactic scale have not been sufficiently investigated yet despite of a large amount of new observational data. The relevant data are provided mainly by extensive surveys of HMSF signposts (water masers, strong FIR sources, ultracompact $\mathrm{H}$ II regions) in lines of high density tracers like CS (Anglada et al. 1996; Bronfman et al. 1996; Juvela 1996; Plume et al. 1992, 1997; Zinchenko et al. 1995, hereafter Paper I). Unfortunately (though inevitably), most of these surveys are limited to one-point observations towards the indicators mentioned above.

In Paper I we started a survey of southern $\mathrm{H}_{2} \mathrm{O}$ and $\mathrm{OH}$ non-stellar masers in the $\mathrm{CS}$ and $\mathrm{C}^{34} \mathrm{~S} J=2-1$ lines with SEST. This survey covered 30 objects in the longitude range $l=260^{\circ}-310^{\circ}$. All detected sources were mapped in the CS line. From the analysis of these data we obtained statistical distributions of the core parameters (Zinchenko 1995, hereafter Paper II). One of the most interesting results was a hint on the galactic gradient of the mean core density.

The goal of the present study is to extend this survey to the outer Galaxy in order to improve the statistics and 
to investigate possible gradients of the core parameters in a larger interval of galactocentric distances.

We use the same selection criteria as in Paper I and the same observing and data analysis procedures. The observations were performed with the $20-\mathrm{m}$ Onsala radio telescope. The difference in the beam sizes of Onsala and SEST is not very significant and has been approximately accounted for by using a simple deconvolution procedure.

In this paper we present the results of our $J=2-1$ $\mathrm{CS}$ and $\mathrm{C}^{34} \mathrm{~S}$ as well as $J=1-0 \mathrm{CO}$ observations towards $55 \mathrm{H}_{2} \mathrm{O}$ masers in the longitude range $l=120^{\circ}-210^{\circ}$. We have mapped most of the detected cores in the CS line. The $\mathrm{C}^{34} \mathrm{~S}$ and $\mathrm{CO}$ observations are limited to the positions of the CS emission peaks.

The structure of the paper is as follows: In Sect. 2 we describe the observations and data analysis. In Sect. 3 we present the observational results. In Sect. 4 we derive some physical parameters of the sources and discuss their properties. In Sect. 5 we present our conclusions.

\section{Observations and data analysis}

\subsection{Observational procedure}

The observations were performed in February 1996 with the 20-m radio telescope of Onsala Space Observatory in Sweden. The half-power beamwidth of the telescope is $40^{\prime \prime}$ at the $\mathrm{CS}$ and $\mathrm{C}^{34} \mathrm{~S}$ frequencies $(97981.00 \mathrm{MHz}$ and $96412.98 \mathrm{MHz}$, respectively) and $35^{\prime \prime}$ at the CO frequency $(115.3 \mathrm{GHz})$. The front-end was a $3 \mathrm{~mm}$ SIS receiver. The single side band system temperature referred to outside the atmosphere was $400-1000 \mathrm{~K}$ for CS, $200-300 \mathrm{~K}$ for $\mathrm{C}^{34} \mathrm{~S}$ and $900-1900 \mathrm{~K}$ for $\mathrm{CO}$ observations depending on the weather conditions and source elevation. The backend was a 256 channel filterbank with $250 \mathrm{kHz}$ resolution.

The observations were made mainly in the frequency switching mode with the frequency offset of $15 \mathrm{MHz}$. The CS spectra at the peak positions and a part of the $\mathrm{C}^{34} \mathrm{~S}$ spectra were obtained using dual beam switching with a beam throw of 11 '.5. Initially, $3 \times 3$ point CS maps with $40^{\prime \prime}$ spacing were obtained for all sources. Then, the mapping was continued according to the initial results and extended normally to $\sim 10 \%$ of the peak antenna temperature value. Pointing was checked periodically by observations of nearby $\mathrm{SiO}$ masers; the pointing accuracy was $\sim 5^{\prime \prime}$.

The standard chopper-wheel technique was used for the calibration. We express the results in the units of main beam brightness temperature $\left(T_{\mathrm{mb}}\right)$ assuming the main beam efficiency $\left(\eta_{\mathrm{mb}}\right)$ of 0.56 and 0.50 at the CS and $\mathrm{CO}$ frequencies, respectively (L.E.B. Johansson, private communication). The temperature scale was checked by observations of Orion A, which we observed also at SEST (Paper I). The comparison with the SEST data shows that the main beam temperatures in these lines agree to within a few percent at the two instruments (we have taken into account the different spectral resolutions in our SEST and Onsala observations).

\subsection{Source list}

Our source list is based on the Arcetri Atlas of galactic $\mathrm{H}_{2} \mathrm{O}$ masers (Comoretto et al. 1990; Palagi et al. 1993; Brand et al. 1994), which covers the declination range $\delta>-30^{\circ}$. The present observations were limited to the longitude range $l=120^{\circ}-212^{\circ}$ (approximately) and $\delta>0$. We managed to observe almost all masers from this atlas satisfying these criteria; their coordinates are given in Table 1. A few objects (S 199, S 255) observed earlier at Metsähovi (Zinchenko et al. 1994) were omitted from the current list. NGC 281 was observed in $\mathrm{C}^{34} \mathrm{~S}$ only since it was not detected in this line at Metsähovi.

We designate all the sources according to their galactic coordinates. The common identifications with some well known objects are given in the last column.

\subsection{Association with the IRAS sources}

We searched the IRAS point source catalogue for possible associations with our targets. In Table 2 (available only electronically) we list the sources from this catalogue located within $5^{\prime}$ from the maser positions. They are present in all cases except G 145.39+4.00. In Cols. (3)-(6) we give the angular offsets of the IRAS sources from the positions listed in Table 1, their coordinates and the IRAS names. In the following columns the flux densities in the four IRAS bands and their quality flags are presented.

Probably not all of these sources are physically associated with the CS cores. However, in all cases where we obtained the CS maps, there are IRAS point sources located within the regions of the CS emission (Sect. 3). We consider this spatial coincidence as a sufficient indication of the physical relationship. In the other cases, where the CS maps have not been obtained, there are IRAS sources within $\sim 1^{\prime}$ from the maser positions. Most probably they are physically related to the CS cores.

\subsection{Data reduction and analysis}

The data were reduced and analyzed in the same way as in Paper I. Briefly, the procedure was the following.

We have reduced the data and produced maps using the GAG (Groupe d'Astrophysique de Grenoble) software package. The measured spectra were fitted by one or more Gaussian components.

The next step in the data analysis was the derivation of the beam-averaged $\mathrm{C}^{34} \mathrm{~S}$ column densities when possible. We have performed it in the LTE approximation. 
Table 1. Source list

\begin{tabular}{|c|c|c|c|}
\hline Name & $\begin{array}{l}\alpha(1950) \\
\left({ }^{\mathrm{h}}\right)\left({ }^{\mathrm{m}}\right)\left({ }^{\mathrm{s}}\right)\end{array}$ & $\begin{array}{l}\delta(1950) \\
\left({ }^{\circ}\right)\left(^{\prime}\right)\left(\left(^{\prime \prime}\right)\right.\end{array}$ & Remarks \\
\hline G $120.15+3.38$ & 002109.6 & 654926 & \\
\hline G $121.30+0.66$ & 003353.3 & 631232 & RNO 1B \\
\hline G $121.37+1.24$ & 003416.1 & 634730 & \\
\hline G $121.38+1.23$ & 003417.2 & 634705 & \\
\hline G $121.74+0.23$ & 003758.7 & 624821 & \\
\hline G $122.78+2.86$ & 004651.2 & 652719 & \\
\hline G $123.07-6.31$ & 004929.2 & 561736 & NGC 281 \\
\hline G $124.64+2.54$ & 010435.7 & 650521 & \\
\hline G $125.52+2.03$ & 011224.5 & 643048 & \\
\hline G $125.63+2.02$ & 011328.2 & 642954 & \\
\hline G $126.68-0.83$ & 011958.0 & 613308 & S 187 \\
\hline G $133.69+1.22$ & 022140.8 & 615326 & W3 (1) \\
\hline G $133.71+1.22$ & 022153.1 & 615222 & W3 (2) \\
\hline G $133.75+1.20$ & 022206.1 & 615140 & W3 (3) \\
\hline G $133.95+1.07$ & $\begin{array}{lll}02 & 23 & 17.3\end{array}$ & 613858 & W3 (OH) \\
\hline G $134.83+1.31$ & 023056.4 & 613315 & \\
\hline G $135.28+2.80$ & 023931.0 & $6244 \quad 16$ & \\
\hline G $135.89-0.46$ & 023320.5 & 593037 & \\
\hline G $136.38+2.27$ & 024611.7 & 614734 & \\
\hline G $137.07+3.00$ & 025411.2 & 620829 & \\
\hline G $138.50+1.64$ & 025922.4 & 601612 & S 201 \\
\hline G $139.91+0.20$ & 030333.2 & 581921 & \\
\hline G $140.64+0.68$ & 031011.7 & 582200 & \\
\hline G $142.00+1.83$ & 032341.0 & 583652 & GL 490 \\
\hline G $143.83-1.56$ & 032111.8 & 544651 & \\
\hline G $144.89+0.45$ & 033520.6 & 554952 & \\
\hline G $145.39+4.00$ & 035424.6 & 581921 & \\
\hline G $148.04+0.63$ & 035326.9 & 540203 & \\
\hline G $149.43+2.06$ & 040700.7 & 541143 & \\
\hline G $150.86+2.19$ & 041438.4 & 531845 & \\
\hline G $151.61-0.46$ & 040624.7 & 505208 & S 209 \\
\hline G $154.35+2.59$ & 043224.0 & 510600 & \\
\hline G $158.04-21.41$ & 032231.1 & 303450 & \\
\hline G $158.35-20.56$ & 032556.6 & 310519 & \\
\hline G $158.76-21.58$ & 032433.9 & 300236 & \\
\hline G $160.26+0.85$ & 044800.0 & 453031 & BFS 44 \\
\hline G $160.51-9.80$ & 040720.1 & 375959 & \\
\hline G $173.48+2.45$ & 053551.3 & 354416 & S 231 \\
\hline G $173.58+2.44$ & 053606.3 & 353921 & \\
\hline G $173.72+2.70$ & 053731.8 & 354018 & S 235 \\
\hline G $180.87+4.09$ & 060040.9 & 301454 & S 241 \\
\hline G $183.72-3.66$ & 053721.8 & 234924 & \\
\hline G $184.96-0.85$ & 055041.0 & 241411 & \\
\hline G $188.95+0.89$ & 060553.7 & 213909 & S 247 \\
\hline G $189.78+0.34$ & 060536.5 & 203934 & S $252 \mathrm{~A}$ \\
\hline G $192.98+0.15$ & 061129.0 & 174609 & \\
\hline G $195.82-0.21$ & 061550.0 & 150609 & \\
\hline G $195.82-0.57$ & 061432.8 & 145547 & S 266 \\
\hline G $196.16-1.25$ & $06 \quad 1244.0$ & 141812 & S 269 B \\
\hline G 196.45-1.68 & 061146.3 & 135031 & S 269 \\
\hline G $197.14-3.10$ & 060801.0 & 123325 & S 270 \\
\hline G $203.32+2.06$ & 063825.5 & 093212 & NGC 2264 \\
\hline G 205.11-14.1 & 054431.3 & 002048 & NGC 2071 \\
\hline G $210.79-2.54$ & 063554.6 & 004720 & S 283 \\
\hline G $211.89-1.23$ & 064236.0 & 002500 & S 284 \\
\hline G 212.25-1.10 & 064341.7 & 000934 & \\
\hline
\end{tabular}

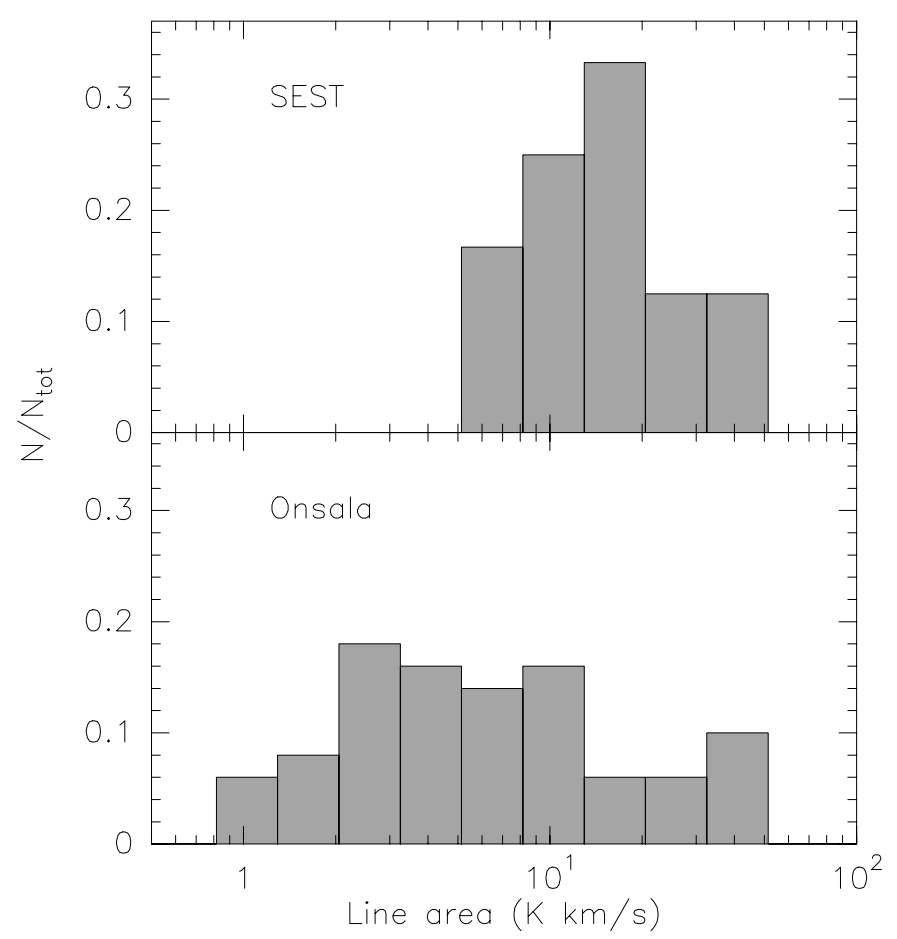

Fig. 1. Histograms of the CS integrated line intensity distributions for the present Onsala sample and for the SEST sample from Paper I

The column densities were found from the $\mathrm{C}^{34} \mathrm{~S}$ line intensity integral assuming for the excitation temperature the value $T_{\text {ex }}=10 \mathrm{~K}$ (the dependence of the $N_{\mathrm{L}}(\mathrm{CS})$ estimates on the assumed $T_{\mathrm{ex}}$ is rather weak; if $T_{\mathrm{ex}}$ increases to $20 \mathrm{~K}$ these estimates change by $35 \%$ ).

We have calculated kinematic distances to our sources from the CS velocities according to the recommendations of Fich et al. (1989), i.e. for a flat rotation curve with $\Theta_{0}=220 \mathrm{~km} \mathrm{~s}^{-1}$ assuming a standard IAU value for $R_{0}$ of $8.5 \mathrm{kpc}$. For some sources no solution could be found in the framework of this model.

The sizes, LTE masses, virial masses and mean densities were calculated as in Paper I using either these kinematic distances or, when available, spectrophotometric distances to the exciting stars of nearby $\mathrm{H}$ II regions. For Gaussian brightness distribution our procedure gives the size at the $\mathrm{e}^{-1}$ level. As in Paper II we use "deconvolved" sizes, being defined as $\theta_{\text {deconv }}=\sqrt{\theta^{2}-\theta_{\mathrm{A}}^{2}}$ where $\theta_{\mathrm{A}}$ is the beam width at the same $\left(\mathrm{e}^{-1}\right)$ level. Of course, this procedure accounts for the beam size only approximately because many cores are elongated or have rather complex brightness distribution. The density estimates have been corrected accordingly.

\section{Observational results}

We detected 47 CS emitting cores in total associated probably with 50 masers. No CS emission was found towards 
Table 3. $\mathrm{CS}$ and $\mathrm{C}^{34} \mathrm{~S}$ line areas and Gaussian line parameters at the indicated positions of the sources. The numbers in the brackets are the statistical uncertainties in the last digits (standard deviations). The upper limits represent the $3 \sigma$ values ( $\sigma$ is rms noise in channels)

\begin{tabular}{|c|c|c|c|c|c|c|c|c|c|c|}
\hline \multirow[b]{2}{*}{ Source } & \multicolumn{6}{|c|}{$\mathrm{CS}$} & \multicolumn{4}{|l|}{$\mathrm{C}^{34} \mathrm{~S}$} \\
\hline & $\begin{array}{l}\Delta \alpha \\
\left({ }^{\prime \prime}\right)\end{array}$ & $\begin{array}{l}\Delta \delta \\
\left({ }^{\prime \prime}\right)\end{array}$ & 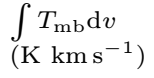 & $\begin{array}{l}T_{\mathrm{mb}} \\
(\mathrm{K})\end{array}$ & $\begin{array}{l}V_{\mathrm{LSR}} \\
\left(\mathrm{km} \mathrm{s}^{-1}\right)\end{array}$ & $\begin{array}{l}\Delta V \\
\left(\mathrm{~km} \mathrm{~s}^{-1}\right)\end{array}$ & $\begin{array}{l}\int T_{\mathrm{mb}} \mathrm{d} v \\
\left(\mathrm{~K} \mathrm{~km} \mathrm{~s}^{-1}\right)\end{array}$ & $\begin{array}{l}T_{\mathrm{mb}} \\
(\mathrm{K})\end{array}$ & $\begin{array}{l}V_{\mathrm{LSR}} \\
\left(\mathrm{km} \mathrm{s}^{-1}\right)\end{array}$ & $\begin{array}{l}\Delta V \\
\left(\mathrm{~km} \mathrm{~s}^{-1}\right)\end{array}$ \\
\hline G $120.15+3.38$ & 0 & 0 & $6.04(29)$ & $2.06(10)$ & $-68.89(06)$ & $2.80(15)$ & $0.42(04)$ & $0.18(02)$ & $-68.87(11)$ & $2.10(30)$ \\
\hline G $121.30+0.66$ & 0 & 0 & $20.16(50)$ & $4.49(13)$ & $-17.71(05)$ & $3.94(13)$ & $3.81(15)$ & $0.81(03)$ & $-17.60(08)$ & $4.20(22)$ \\
\hline G $121.37+1.24$ & 0 & 40 & $4.29(27)$ & $1.01(07)$ & $-28.51(12)$ & $3.98(31)$ & & $<0.15$ & & \\
\hline G $121.74+0.23$ & 0 & 0 & $5.98(26)$ & $2.01(12)$ & $-17.76(05)$ & $2.35(16)$ & $1.01(10)$ & $0.23(02)$ & $-16.69(20)$ & $4.11(49)$ \\
\hline G $122.78+2.86$ & 0 & 0 & $2.51(16)$ & $1.00(07)$ & $-63.43(07)$ & $2.26(19)$ & $0.24(05)$ & $0.18(02)$ & $-62.98(14)$ & $1.46(23)$ \\
\hline G $123.07-6.31$ & & & & & & & $2.21(14)$ & $0.54(04)$ & $-30.38(11)$ & $3.85(31)$ \\
\hline G $124.64+2.54$ & 0 & 0 & $2.14(18)$ & $0.56(04)$ & $-86.54(15)$ & $3.67(34)$ & & & & \\
\hline G $125.52+2.03$ & 0 & 0 & $3.09(21)$ & $0.98(08)$ & $-54.50(09)$ & $2.71(26)$ & & $<0.10$ & & \\
\hline G $125.63+2.02$ & 0 & 0 & $1.90(14)$ & $0.51(04)$ & $-54.61(12)$ & $3.38(36)$ & & & & \\
\hline G $126.68-0.83$ & 160 & 0 & $10.78(23)$ & $4.29(10)$ & $-14.26(02)$ & $2.30(06)$ & $2.63(08)$ & $1.00(04)$ & $-14.05(03)$ & $2.25(10)$ \\
\hline G $133.69+1.22$ & 0 & -40 & $47.18(49)$ & $8.82(10)$ & $-43.09(02)$ & $4.76(06)$ & & & & \\
\hline G $133.95+1.07$ & 0 & 0 & $38.54(49)$ & $6.83(08)$ & $-47.69(03)$ & $5.36(07)$ & $8.79(26)$ & $1.49(05)$ & $-47.17(08)$ & $5.44(20)$ \\
\hline G $134.83+1.31$ & 0 & 40 & $4.21(37)$ & $1.30(14)$ & $-39.55(12)$ & $2.79(34)$ & & $<0.16$ & & \\
\hline G $135.28+2.80$ & 0 & 0 & $3.80(18)$ & $0.88(04)$ & $-71.26(09)$ & $4.01(23)$ & $0.81(13)$ & $0.25(04)$ & $-71.74(23)$ & $3.03(62)$ \\
\hline G $135.89-0.46$ & -40 & -40 & $1.68(34)$ & $0.45(07)$ & $-25.54(40)$ & $4.08(74)$ & & & & \\
\hline G $136.38+2.27$ & 0 & 0 & $4.52(23)$ & $1.24(08)$ & $-42.15(08)$ & $3.03(23)$ & $0.69(10)$ & $0.16(02)$ & $-42.39(28)$ & $4.15(85)$ \\
\hline G $137.07+3.00$ & 0 & 0 & $2.80(11)$ & $0.78(03)$ & $-52.19(06)$ & $3.47(16)$ & $0.74(13)$ & $0.19(03)$ & $-53.01(29)$ & $4.52(85)$ \\
\hline G $138.50+1.64$ & 0 & 0 & $2.42(19)$ & $0.77(07)$ & $-38.16(11)$ & $2.81(29)$ & & $<0.20$ & & \\
\hline G $139.91+0.20$ & 0 & 0 & $10.84(24)$ & $3.87(09)$ & $-39.50(02)$ & $2.61(07)$ & $0.86(11)$ & $0.34(04)$ & $-39.58(16)$ & $2.51(34)$ \\
\hline G $140.64+0.67$ & 0 & 0 & $6.07(23)$ & $2.22(11)$ & $-38.26(04)$ & $2.22(13)$ & $0.69(14)$ & $0.16(03)$ & $-38.49(44)$ & $4.21(95)$ \\
\hline G $142.00+1.83$ & 0 & 0 & $6.48(45)$ & $2.27(18)$ & $-13.22(09)$ & $2.49(22)$ & $0.25(06)$ & $0.12(03)$ & $-12.89(26)$ & $1.99(57)$ \\
\hline G $143.83-1.56$ & 0 & 0 & $0.93(23)$ & $0.19(04)$ & $-23.28(59)$ & $4.19(114)$ & & & & \\
\hline G $150.86+2.19$ & 0 & -40 & $1.86(54)$ & $0.45(09)$ & $-39.64(57)$ & $5.61(129)$ & & & & \\
\hline G $151.61-0.46$ & 0 & 0 & $2.97(18)$ & $0.67(06)$ & $-49.45(12)$ & $2.97(29)$ & & $<0.20$ & & \\
\hline G $154.35+2.59$ & 0 & 0 & $9.05(22)$ & $2.67(08)$ & $-36.60(03)$ & $2.97(09)$ & & $<0.18$ & & \\
\hline G $158.04-21.40$ & 0 & 0 & $6.20(20)$ & $2.72(10)$ & $4.43(03)$ & $1.96(08)$ & & & & \\
\hline G $158.35-20.60$ & 0 & 0 & $11.32(26)$ & $3.79(11)$ & $8.08(03)$ & $2.47(07)$ & & $<0.20$ & & \\
\hline G $158.76-21.60$ & 0 & 0 & $3.45(14)$ & $1.32(05)$ & $5.15(05)$ & $2.62(11)$ & & $<0.14$ & & \\
\hline G $160.26+0.85$ & 0 & 0 & $3.87(17)$ & $1.29(05)$ & $-25.50(06)$ & $2.77(14)$ & & & & \\
\hline G $160.51-9.80$ & 0 & 0 & $1.78(12)$ & $1.19(09)$ & $-3.77(05)$ & $1.26(10)$ & & & & \\
\hline G $173.48+2.45$ & 0 & 0 & $27.19(30)$ & $4.40(05)$ & $-16.62(03)$ & $5.55(07)$ & $2.99(13)$ & $0.69(03)$ & $-16.74(08)$ & $3.95(22)$ \\
\hline G $173.58+2.44$ & 17 & -25 & $13.72(85)$ & $2.78(27)$ & $-16.62(12)$ & $3.78(42)$ & & & & \\
\hline G $173.72+2.70$ & 0 & 0 & $20.67(28)$ & $6.14(09)$ & $-17.03(02)$ & $3.07(05)$ & $4.18(10)$ & $1.38(04)$ & $-17.12(03)$ & $2.69(09)$ \\
\hline G $180.87+4.09$ & 0 & 0 & $9.85(24)$ & $2.47(08)$ & $-8.69(04)$ & $3.03(12)$ & $1.47(13)$ & $0.44(04)$ & $-8.87(14)$ & $2.96(35)$ \\
\hline G $183.72-3.66$ & 0 & 0 & $11.19(33)$ & $2.24(07)$ & $2.00(06)$ & $4.36(17)$ & & $<0.24$ & & \\
\hline G $184.96-0.85$ & 0 & 0 & $1.06(22)$ & $0.36(10)$ & $6.19(28)$ & $2.26(73)$ & $0.63(12)$ & $0.26(05)$ & $5.56(23)$ & $2.27(50)$ \\
\hline G $188.95+0.89$ & 0 & 0 & $38.03(49)$ & $8.07(12)$ & $3.30(02)$ & $3.90(06)$ & $2.76(15)$ & $0.69(05)$ & $3.13(09)$ & $3.37(28)$ \\
\hline G $189.78+0.34$ & 0 & 0 & $18.85(28)$ & $4.42(07)$ & $9.12(02)$ & $3.64(07)$ & $2.38(14)$ & $0.65(04)$ & $8.91(10)$ & $3.30(24)$ \\
\hline G $192.98+0.15$ & 0 & 0 & $8.18(54)$ & $2.76(18)$ & $8.48(09)$ & $2.85(21)$ & & & & \\
\hline G $195.82-0.21$ & 0 & -80 & $14.73(243)$ & $2.16(33)$ & $32.89(56)$ & $6.83(118)$ & & & & \\
\hline G $195.82-0.57$ & 0 & 0 & $4.59(55)$ & $0.90(13)$ & $33.50(28)$ & $4.07(66)$ & & & & \\
\hline G $196.16-1.25$ & 0 & 0 & $2.28(38)$ & $0.81(14)$ & $12.32(22)$ & $2.43(48)$ & & & & \\
\hline G $196.45-1.68$ & 0 & 0 & $7.82(67)$ & $1.63(15)$ & $18.15(18)$ & $4.44(46)$ & & & & \\
\hline G $197.14-3.10$ & 80 & -40 & $3.24(139)$ & $1.51(49)$ & $23.46(44)$ & $2.47(90)$ & & & & \\
\hline G $203.32+2.06$ & 0 & 0 & $36.93(96)$ & $7.05(19)$ & $8.08(06)$ & $4.86(15)$ & & & & \\
\hline G 205.11-14.10 & 0 & 0 & $32.41(75)$ & $4.76(16)$ & $9.78(06)$ & $5.22(20)$ & $0.96(13)$ & $0.22(03)$ & $9.96(28)$ & $4.14(67)$ \\
\hline G $210.79-2.54$ & 0 & 40 & $2.77(55)$ & $0.71(14)$ & $46.66(37)$ & $3.47(77)$ & & & & \\
\hline G $212.25-1.10$ & 0 & 0 & $2.53(28)$ & $0.64(07)$ & $41.90(20)$ & $3.61(49)$ & & & & \\
\hline
\end{tabular}

5 masers. The $\mathrm{C}^{34} \mathrm{~S}$ emission was detected in 19 of these cores (some of them just have not been observed in the $\mathrm{C}^{34} \mathrm{~S}$ line). In addition, $\mathrm{C}^{34} \mathrm{~S}$ was detected in NGC 281. So the CS detection rate is comparable to that achieved at SEST (Paper I). However, the CS emission for the present sample is in general much weaker than for the SEST sample. This fact is illustrated by Fig. 1 where we plot the histograms of the integrated line intensity distributions for both samples in the same temperature scale. The possible influence of the difference in the beam size could only smoothen the difference in the line intensities. It is worth noting that the average distances to the sources in both samples are similar.
The sources studied in the present work are located in the outer Galaxy while most SEST sources belong to the inner Galaxy. Thus, Fig. 1 clearly shows a difference between them.

Due to the relative weakness of these sources we were unable to obtain the same set of data for each source as in Paper I. Only for about a third of the detected cores (15) do we have a comparable amount of data (reasonably complete CS map, $\mathrm{C}^{34} \mathrm{~S}$ and $\mathrm{CO}$ observations near the peaks). We shall refer to these sources as sources of category I. For 11 other cores the CS maps can be considered to be complete, but an absence or low quality of the $\mathrm{C}^{34} \mathrm{~S}$ data do not enable us to estimate masses and densities in the same way as for the first category sources. These objects will be 
referred to as sources of category II. For the remaining 21 sources (category III) the data are incomplete and we cannot derive sizes, masses, virial masses and densities. The physical information obtained for these sources comprises mostly the kinetic temperature (from the $\mathrm{CO}$ brightness), velocity dispersion (from the line widths) and kinematic distance.

In Table 3 we present the $\mathrm{CS}$ and $\mathrm{C}^{34} \mathrm{~S}$ integrated line intensities and Gaussian line parameters for detected sources. These data refer either to the grid positions closest to the CS emission peaks or to the $(0,0)$ positions. A typical rms noise for non-detected sources is $\sim 0.1-0.2 \mathrm{~K}$ in $250 \mathrm{kHz}$ channels.

For the sources of the categories I and II we present also the CS maps and the CO, CS and, when available, $\mathrm{C}^{34} \mathrm{~S}$ spectra at the indicated positions (Fig. 2).

\section{Discussion}

\subsection{What is a core?}

An important question to start with is how to define a core? In Papers I and II we assumed implicitly that a core is a region of noticeable CS emission towards our HMSF pointers (masers). Many of these regions have an apparent substructure; others might reveal it when observed with a higher resolution. Indeed, observations of higher CS transitions (with better angular resolution) by Lapinov et al. (1998) have revealed complex structure of several cores which show almost spherically symmetric appearance in CS $J=2-1$. It might be possible to consider the clumps constituting this substructure as individual cores. However, the internal fragmentation continues probably to smaller and smaller scales and it is not clear where it stops.

Taking this into account we prefer to consider these CS emitting regions as single entities having probably rather complex internal structure. Most of them are concentrated to well defined single emission peaks which coincide with powerful IR sources, masers, etc. Then, the fact that practically all these regions seem to be gravitationally bound (see below) also supports this approach.

\subsection{Physical parameters of the cores}

In Paper II we constructed statistical distributions of the core parameters on the basis of the SEST data. There was a hint of the decrease of the mean core density with the galactocentric radius. The new results presented here enable us to improve these statistics and to investigate further the galactic gradients of these parameters.

First we have to derive the physical parameters of the cores. For the sources of category I we make it in essentially the same way as in Papers I and II as described briefly in Sect. 2.4. The results are presented in Table 4.
In addition, we give the IR luminosities of associated point IRAS sources and their colour temperatures $\left(T_{\mathrm{d}}\right)$ from the ratio of the 60 and $100 \mu \mathrm{m}$ fluxes calculated as in Henning et al. (1990).

For the sources of category II we are unable to derive LTE masses and densities in the same way. However, sizes and virial masses can be determined as for the category I sources. Our previous results (Papers I and II) as well as an inspection of the results for the category I sources show that LTE masses derived from the column densities and virial masses are very close to each other in most cases. So for the sources of category II we estimate the densities from the virial masses. The results of these estimations are presented in Table 5.

The physical parameters determined for the sources of category III are given in Table 6 .

\subsection{Statistical distributions of the parameters}

As in Paper II when constructing statistical distributions of the core parameters we limit ourselves to the objects located within a certain distance from the sun in order to diminish the selection effects. We consider here two subsamples: (1) $1 \mathrm{kpc}<d<4 \mathrm{kpc}$ and (2) $d<5 \mathrm{kpc}$. The comparison of these subsamples shows the influence of the nearest and most distant sources. We combine here our previous SEST and present Onsala data. In total there are 37 cores in the first subsample and 43 in the second one with reliably determined parameters (categories I and II for the Onsala sample).

In addition to the $\mathrm{CO}$ brightness temperature, size, mean density, LTE mass and CS line width as in Paper II we consider here the IR luminosity to mass ratio which characterizes the star formation process. The histograms of the statistical distributions for these parameters are plotted in Fig. 3.

An inspection of these histograms leads to the following conclusions:

The CO brightness temperature distribution peaks at $\sim 20-30 \mathrm{~K}$; for most cores $(\sim 90 \%)$ these temperatures lie in the range $15-50 \mathrm{~K}$. Since the CO transitions are easily thermalized this temperature should be close to the kinetic temperature somewhere in the source (minus the background temperature). However, due to a very high optical depth in $\mathrm{CO}$ lines, the innermost regions can be shielded (if there is no significant velocity gradient or significant clumpiness). Therefore, the kinetic temperatures determined from CO might refer to the outer regions of the cores. It is worth noting, however, that they are rather close to the temperatures found from ammonia observations of HMSF cores in Effelsberg with a similar beam size (Zinchenko et al. 1997) which gave $T_{\text {kin }} \approx 20-30 \mathrm{~K}$ near the peaks of ammonia emission. These temperatures reflect the conditions in the dense gas where ammonia is excited (though averaged over the beam). The CO temperatures are also close to the colour temperatures of the 

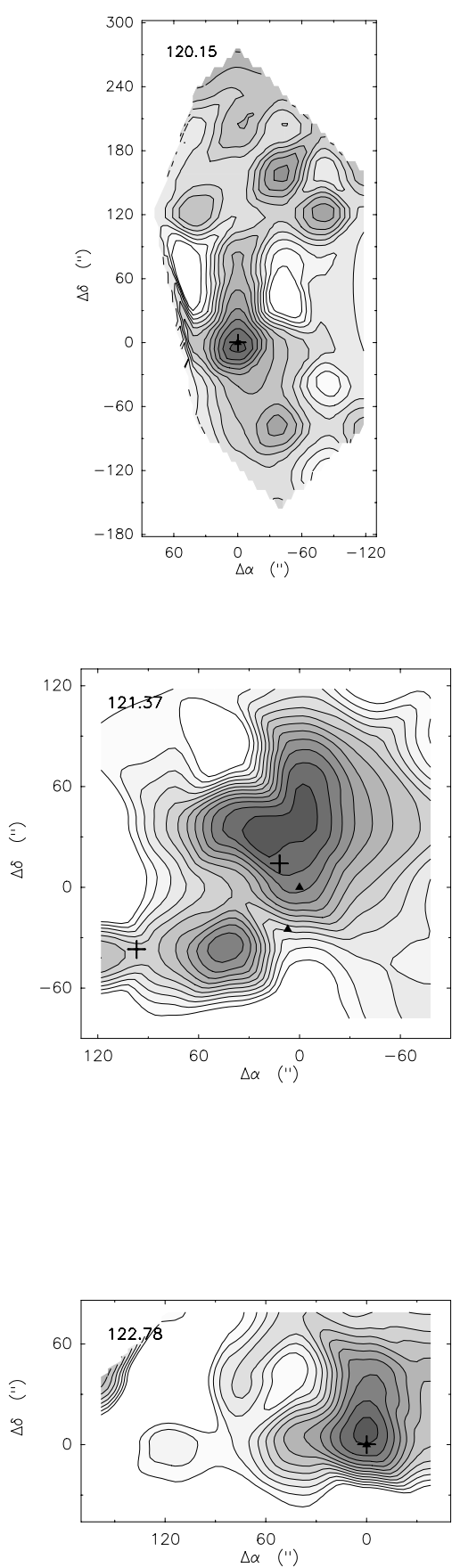
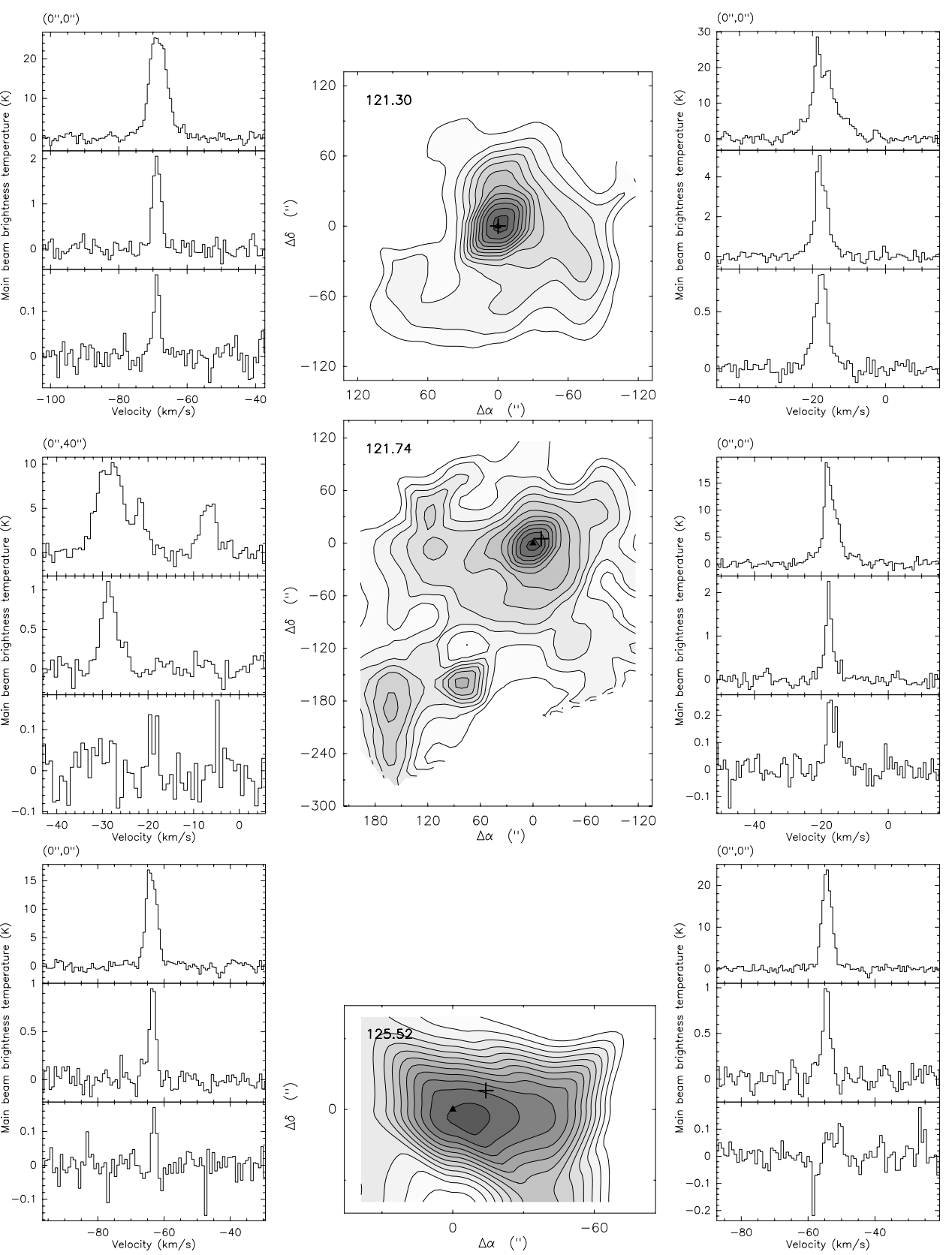

Fig. 2. Left panels: the integrated CS line intensity maps for the sources of categories I and II (see text). The levels start from $15 \%$ of the peak intensities in steps of $7.5 \%$. The peak intensities are nearly equal to those presented in Table 3 . Crosses mark the IRAS positions. Triangles indicate water masers. Right panels: $\mathrm{CO}, \mathrm{CS}$ and $\mathrm{C}^{34} \mathrm{~S}$ spectra at the indicated positions

embedded IRAS sources determined from the ratio of the 60 and $100 \mu \mathrm{m}$ fluxes (Tables 4-6), i.e. the gas kinetic temperature is close to the temperature of cold dust component which emits at these wavelengths.

We present the size, mass and density distributions in the way which is typical for mass distribution, i.e. by the number density per unit (linear) interval of the parameter. This function for mass is called the mass spectrum and we can talk analogously about size and density spectra. Most cores have sizes of $1.0-1.5$ pc. There is no cores with $L \gtrsim 2.5 \mathrm{pc}$ in the considered subsamples. It means in particular that the angular size of a typical core equals our beam size at the distance of $3-5 \mathrm{kpc}$ which supports our selection of the distance limits since many of more distant cores would be unresolved.

The mean density of the cores is $10^{3}-10^{5} \mathrm{~cm}^{-3}$. We emphasize that this is an average density defined as $\bar{n}=N_{\mathrm{L}}\left(\mathrm{H}_{2}\right) / L$. This density is, at least at the lower 

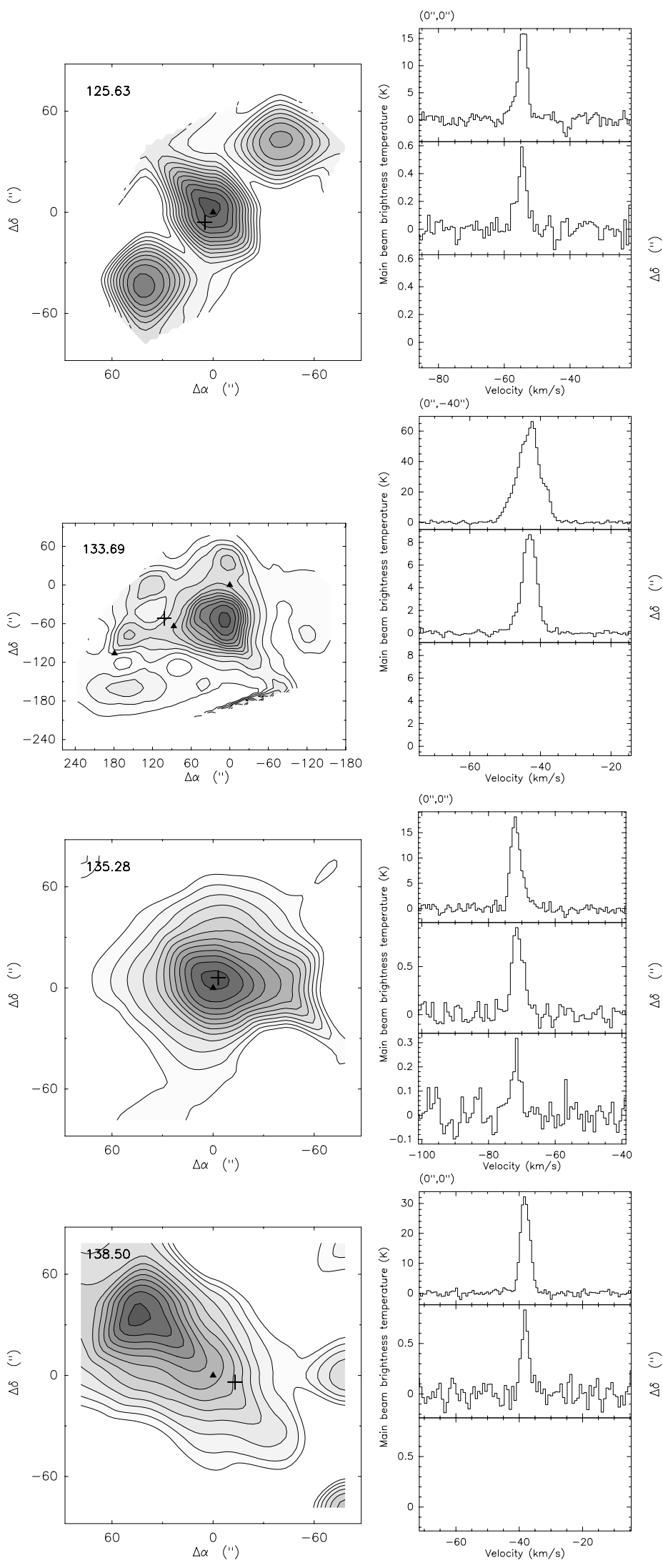

Fig. 2. continued
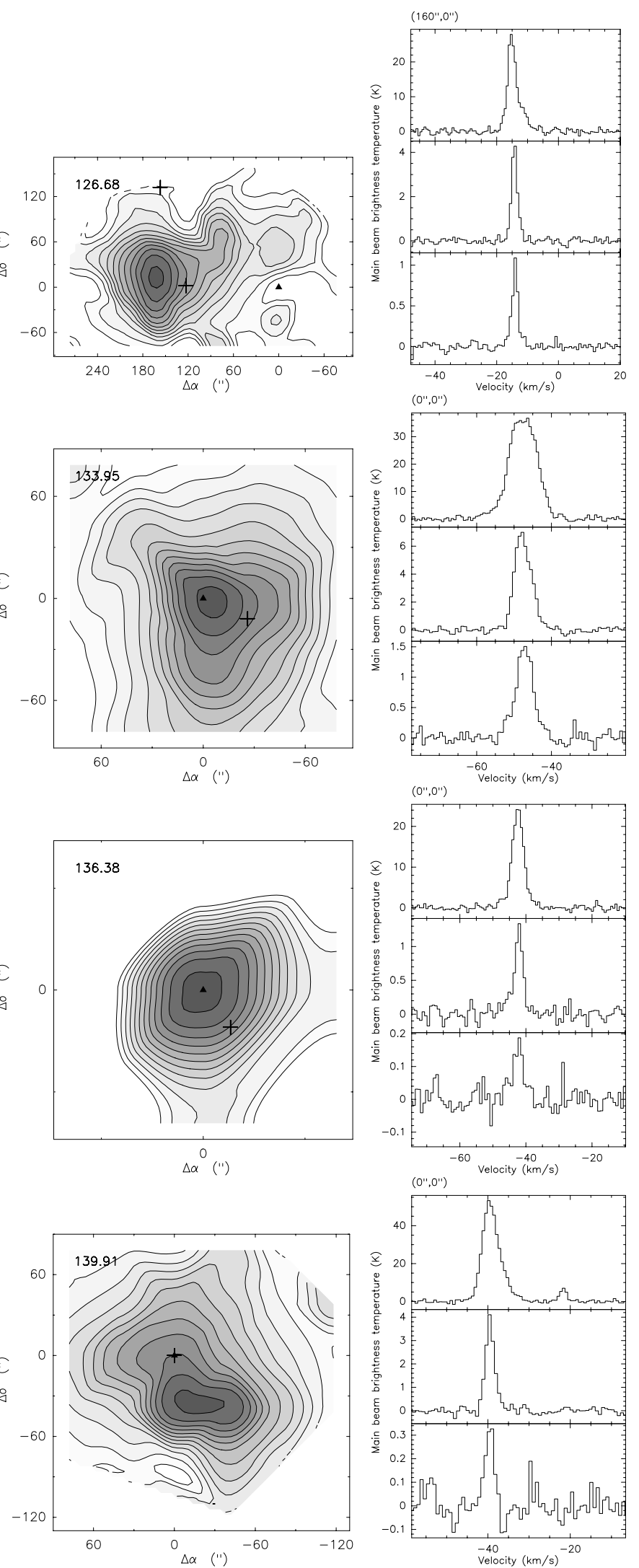

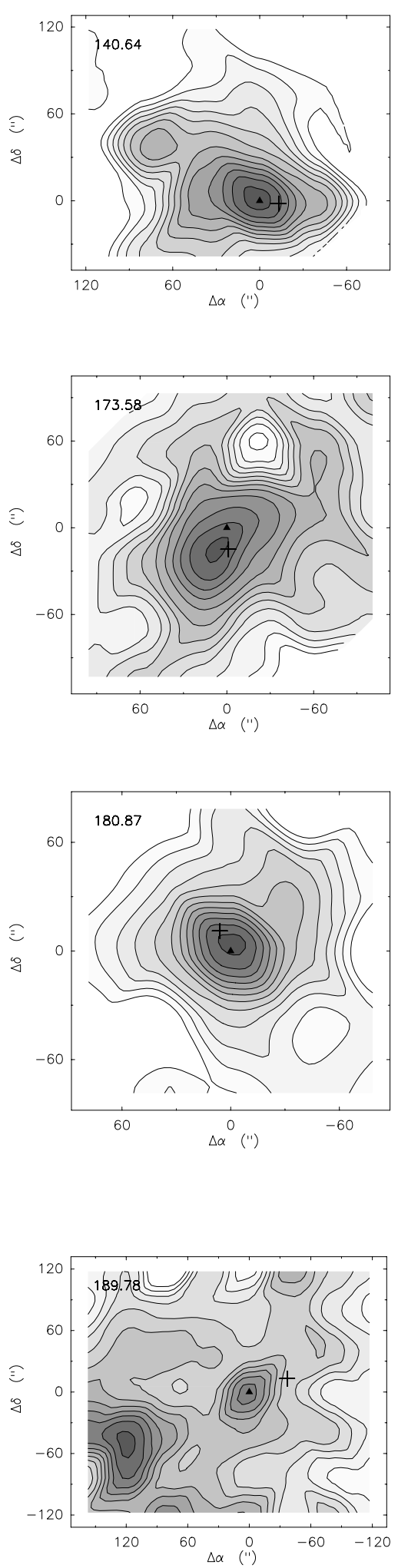

Fig. 2. continued
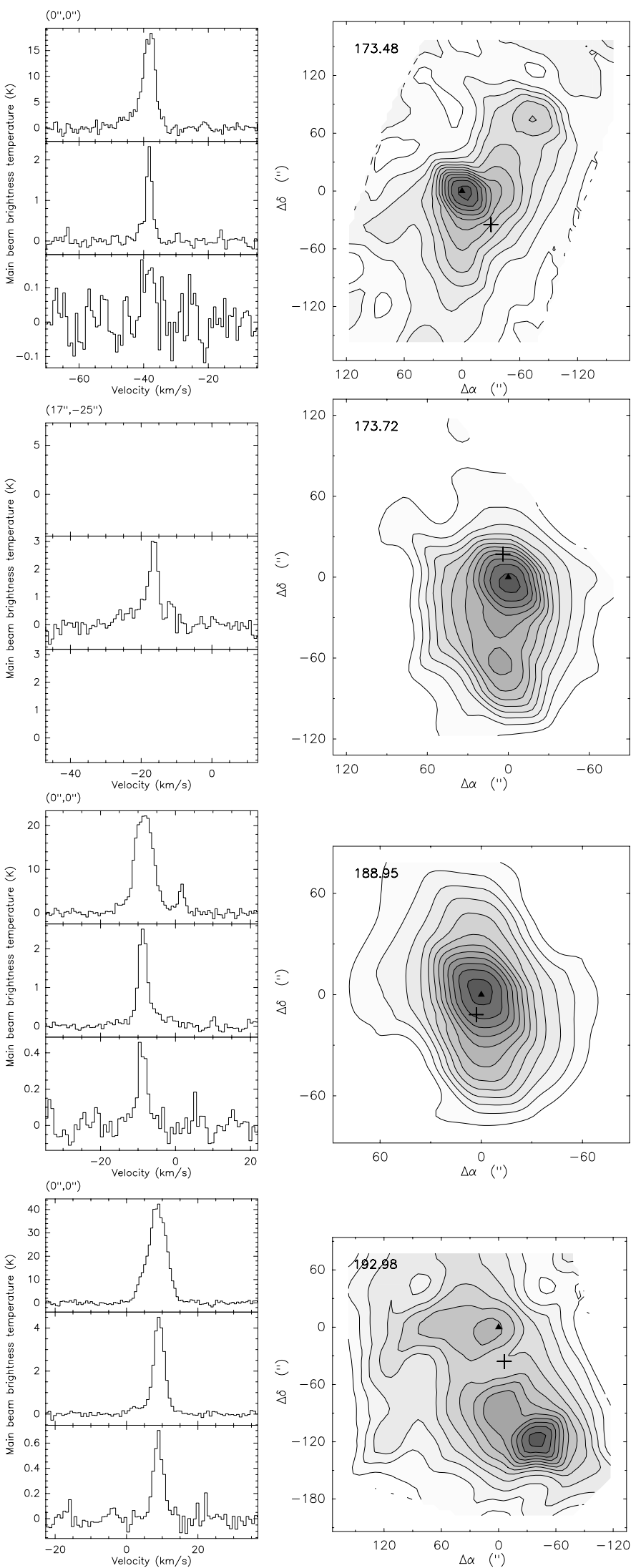
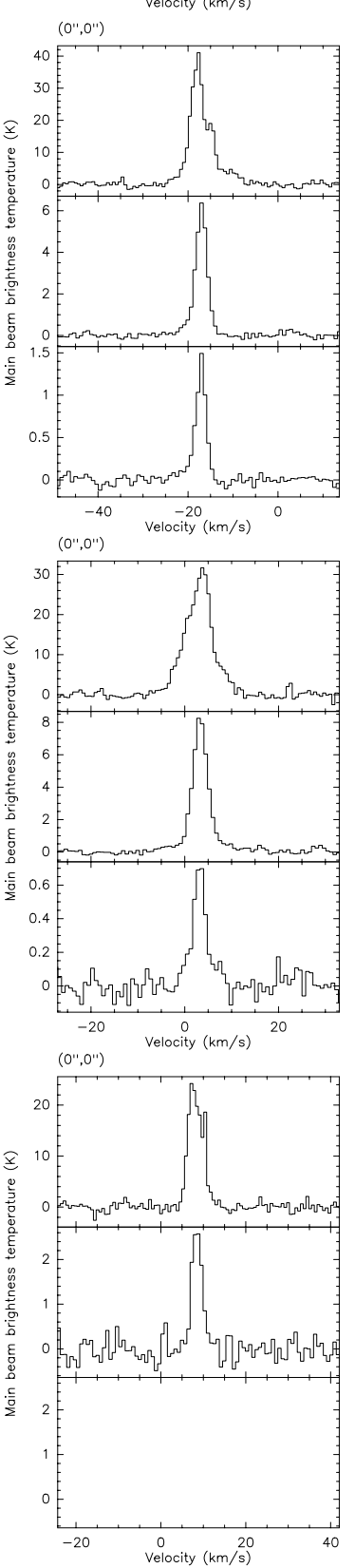

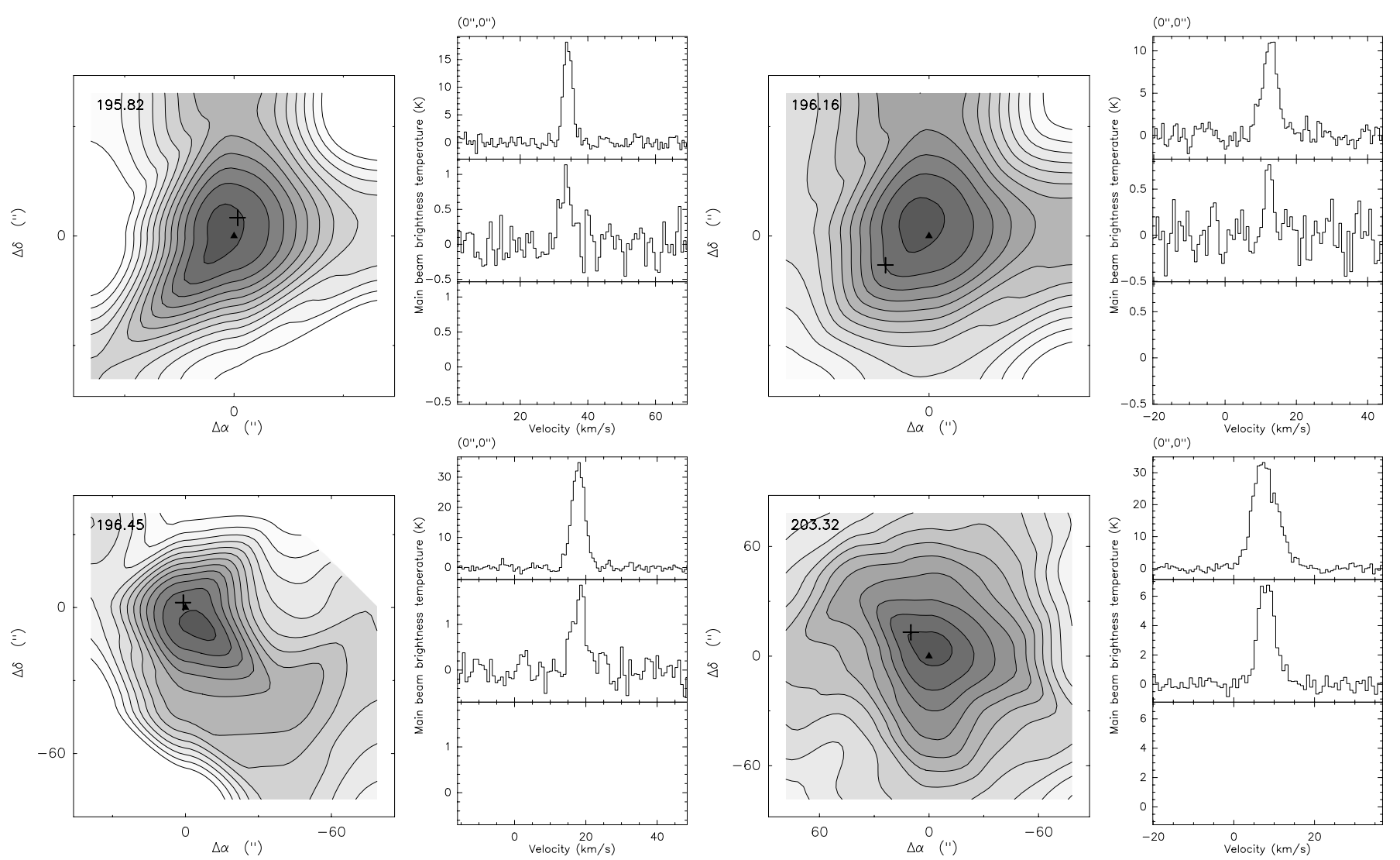

Fig. 2. continued

Table 4. Physical parameters of the sources of category I

\begin{tabular}{|c|c|c|c|c|c|c|c|c|c|c|c|}
\hline Name & $\begin{array}{r}d \\
(\mathrm{kpc})\end{array}$ & $\begin{array}{r}R \\
(\mathrm{kpc})\end{array}$ & $\begin{array}{r}z \\
(\mathrm{pc})\end{array}$ & $\begin{array}{r}L \\
\text { (pc) }\end{array}$ & $\begin{array}{r}\log \bar{n} \\
\left(\mathrm{~cm}^{-3}\right)\end{array}$ & $\begin{array}{r}M \\
M_{\odot}\end{array}$ & $\begin{array}{r}M_{\text {vir }} \\
M_{\odot}\end{array}$ & $\begin{array}{r}T_{\mathrm{mb}}(\mathrm{CO}) \\
(\mathrm{K})\end{array}$ & $\begin{array}{l}\Delta V(\mathrm{CS}) \\
\left(\mathrm{km} \mathrm{s}^{-1}\right)\end{array}$ & $\begin{array}{r}L_{\mathrm{IR}} \\
\left(L_{\odot}\right)\end{array}$ & $\begin{array}{l}T_{\mathrm{d}} \\
(\mathrm{K})\end{array}$ \\
\hline G $120.15+3.38$ & $6.6^{a}$ & 13.1 & 388 & 6.4 & 2.79 & 8326 & 14595 & 25 & 4.67 & $2.010^{4}$ & 39 \\
\hline G $121.30+0.66$ & $0.9^{b}$ & 9.0 & 9 & 0.4 & 4.96 & 397 & 339 & 29 & 2.72 & $5.410^{2}$ & 36 \\
\hline G $121.74+0.23$ & $1.3^{a}$ & 9.3 & 5 & 1.3 & 3.85 & 825 & 481 & 18 & 1.87 & $4.510^{1}$ & 32 \\
\hline G $122.78+2.86$ & $5.9^{a}$ & 12.7 & 294 & 2.8 & 2.96 & 1070 & 1579 & 16 & 2.31 & $2.210^{4}$ & 46 \\
\hline G $126.68-0.83$ & $1.0^{a}$ & 9.1 & -13 & 0.8 & 4.48 & 852 & 651 & 28 & 2.76 & $<2.210^{3}$ & \\
\hline G $133.95+1.07$ & $2.1^{c}$ & 10.1 & 39 & 1.3 & 4.85 & 7426 & 2650 & 35 & 4.47 & $7.010^{4}$ & 44 \\
\hline G $135.28+2.80$ & $8.0^{a}$ & 15.2 & 388 & 2.2 & 3.76 & 2988 & 4133 & 18 & 4.27 & $4.710^{4}$ & 50 \\
\hline G $136.38+2.27$ & $3.7^{a}$ & 11.5 & 146 & 0.7 & 4.30 & 397 & 292 & 24 & 1.96 & $1.110^{4}$ & 42 \\
\hline G $139.91+0.20$ & $3.6^{a}$ & 11.5 & 12 & 2.5 & 3.51 & 2778 & 2328 & 47 & 2.95 & $4.110^{4}$ & 43 \\
\hline G $140.64+0.67$ & $3.4^{a}$ & 11.3 & 39 & 1.8 & 3.60 & 1221 & 567 & 18 & 1.73 & $6.910^{2}$ & 31 \\
\hline G $173.48+2.45$ & $2.3^{c}$ & 10.8 & 98 & 1.9 & 4.18 & 5259 & 5063 & 23 & 5.06 & $8.510^{3}$ & 36 \\
\hline G $173.72+2.70$ & $2.3^{c}$ & 10.8 & 108 & 1.2 & 4.54 & 3494 & 984 & 40 & 2.74 & $1.810^{4}$ & 48 \\
\hline G $180.87+4.09$ & $4.7^{c}$ & 13.2 & 335 & 2.2 & 3.88 & 3940 & 4059 & 22 & 4.23 & $<1.110^{4}$ & \\
\hline G $188.95+0.89$ & $3.5^{c}$ & 12.0 & 54 & 1.5 & 4.32 & 3591 & 2264 & 31 & 3.81 & $2.810^{4}$ & 37 \\
\hline G $189.78+0.34$ & $1.5^{c}$ & 10.0 & 8 & 1.7 & 4.12 & 3132 & 3117 & 42 & 4.23 & $4.910^{3}$ & 38 \\
\hline
\end{tabular}

${ }^{a}$ Kinematic; ${ }^{b}$ Yang et al. (1991); ${ }^{c}$ Brand \& Blitz (1993). 
Table 5. Physical parameters of the sources of category II

\begin{tabular}{lrrrrrrrrrr}
\hline Name & $\begin{array}{r}d \\
(\mathrm{kpc})\end{array}$ & $\begin{array}{r}R \\
(\mathrm{kpc})\end{array}$ & $\begin{array}{r}z \\
(\mathrm{pc})\end{array}$ & $\begin{array}{r}L \\
(\mathrm{pc})\end{array}$ & $\begin{array}{r}\log \bar{n} \\
\left(\mathrm{~cm}^{-3}\right)\end{array}$ & $\begin{array}{r}M_{\text {vir }} \\
M_{\odot}\end{array}$ & $T_{\mathrm{mb}}(\mathrm{CO})$ & $\begin{array}{c}\Delta V(\mathrm{CS}) \\
\left(\mathrm{km} \mathrm{s}^{-1}\right)\end{array}$ & $\begin{array}{c}L_{\mathrm{IR}} T_{\mathrm{d}} \\
\left(L_{\odot}\right)\end{array}$ & $(\mathrm{K})$ \\
\hline G 121.37+1.24 & $2.3^{a}$ & 9.9 & 49 & 1.6 & 4.15 & 3604 & 10 & 4.61 & $4.110^{2}$ & 29 \\
G 125.52+2.03 & $4.8^{a}$ & 12.0 & 171 & 1.7 & 3.41 & 1105 & 23 & 2.51 & $1.410^{3}$ & 29 \\
G 125.63+2.02 & $4.9^{a}$ & 12.0 & 171 & 1.7 & 3.69 & 2029 & 16 & 3.42 & $2.910^{3}$ & 39 \\
G 133.69+1.22 & $2.1^{b}$ & 10.1 & 44 & 1.9 & 4.53 & 13271 & 65 & 8.16 & $<2.510^{5}$ & \\
G 138.50+1.64 & $3.3^{a}$ & 11.2 & 94 & 1.7 & 3.63 & 1401 & 31 & 2.82 & $2.610^{4}$ & 36 \\
G 173.58+2.44 & $2.3^{b}$ & 10.8 & 97 & 1.8 & 3.47 & 1098 & & $2.38<4.210^{3}$ & \\
G 192.98+0.15 & $2.5^{b}$ & 11.0 & 6 & 2.3 & 3.47 & 2031 & 24 & 2.91 & $7.010^{3}$ & 42 \\
G 195.82-0.57 & $14.4^{a}$ & 22.7 & -143 & 3.5 & 2.72 & 3214 & 18 & 2.94 & $1.910^{4} 35$ \\
G 196.16-1.25 & $3.8^{b}$ & 12.2 & -82 & 1.4 & 3.66 & 1046 & 11 & 2.69 & $4.310^{3}$ & 43 \\
G 196.45-1.68 & $3.8^{b}$ & 12.2 & -111 & 1.7 & 3.82 & 2405 & 35 & 3.68 & $4.310^{4}$ & 41 \\
G 203.32+2.06 & $1.5^{a}$ & 9.8 & 52 & 1.1 & 4.41 & 1825 & 33 & 4.05 & $7.710^{3}$ & 37 \\
\hline
\end{tabular}

${ }^{a}$ Kinematic; ${ }^{b}$ Brand \& Blitz (1993).

Table 6. Physical parameters of the sources of category III

\begin{tabular}{lrrrrrrr}
\hline Name & $\begin{array}{r}d \\
(\mathrm{kpc})\end{array}$ & $\begin{array}{r}R \\
(\mathrm{kpc})\end{array}$ & $\begin{array}{r}z \\
(\mathrm{pc})\end{array}$ & $T_{\mathrm{mb}}(\mathrm{CO})$ & $\begin{array}{r}\Delta V(\mathrm{CS}) \\
(\mathrm{Km} \mathrm{s})\end{array}$ & $\begin{array}{r}L_{\mathrm{IR}} T_{\mathrm{d}} \\
\left(L_{\odot}\right)\end{array}$ & $(\mathrm{K})$ \\
\hline G 124.64+2.54 & $9.4^{a}$ & 15.9 & 418 & 17 & 3.70 & $6.710^{4}$ & 44 \\
G 134.83+1.31 & $3.3^{a}$ & 11.1 & 76 & 20 & 3.79 & $1.710^{3}$ & 29 \\
G 135.89-0.46 & $1.9^{a}$ & 10.0 & -15 & 24 & 4.08 & $2.410^{2}$ & 34 \\
G 137.07+3.00 & $5.0^{a}$ & 12.7 & 263 & 12 & 3.62 & $3.710^{3}$ & 37 \\
G 142.00+1.83 & $0.8^{a}$ & 9.2 & 26 & 29 & 3.21 & $1.810^{3}$ & 45 \\
G 143.83-1.56 & $1.8^{a}$ & 10.0 & -49 & 20 & 4.19 & $3.710^{3}$ & 37 \\
G 150.86+2.19 & $4.7^{a}$ & 12.8 & 179 & 21 & 5.67 & $2.410^{3}$ & 34 \\
G 151.61-0.46 & $7.1^{a}$ & 15.1 & -56 & 17 & 5.27 & $2.410^{4}$ & 35 \\
G 154.35+2.59 & $4.8^{a}$ & 13.0 & 216 & 18 & 2.49 & $3.410^{4}$ & 47 \\
G 158.04-21.40 & $0.3^{b}$ & 8.8 & -127 & 12 & 1.92 & $1.610^{1}$ & 24 \\
G 158.35-20.60 & $0.3^{b}$ & 8.8 & -123 & 33 & 2.48 & $<1.110^{2}$ & \\
G 158.76-21.60 & $0.3^{b}$ & 8.8 & -128 & 14 & 2.25 & $1.310^{1}$ & 35 \\
G 160.26+0.85 & $3.6^{a}$ & 12.0 & 53 & 15 & 2.64 & $6.610^{2}$ & 30 \\
G 160.51-9.80 & $0.3^{c}$ & 8.8 & -59 & 12 & 1.35 & $2.310^{1}$ & 42 \\
G 183.72-3.66 & $6.5^{a}$ & 15.0 & -416 & 10 & 2.55 & $1.510^{4}$ & 39 \\
G 184.96-0.85 & $10.2^{a}$ & 18.7 & -151 & 8 & 2.26 & $4.810^{4}$ & 48 \\
G 195.82-0.21 & $13.8^{a}$ & 22.1 & -50 & 15 & 3.30 & $5.410^{3}$ & 35 \\
G 197.14-3.10 & $6.4^{a}$ & 14.8 & -347 & 11 & 2.62 & $2.110^{3}$ & 42 \\
G 205.11-14.10 & $0.4^{d}$ & 8.9 & -99 & 30 & 4.84 & $3.510^{2}$ & \\
G 210.79-2.54 & $7.4^{a}$ & 15.4 & -329 & 3 & 3.30 & $8.610^{3}$ & 35 \\
G 212.25-1.10 & $5.9^{a}$ & 13.9 & -113 & 20 & 2.13 & $6.210^{3}$ & 38 \\
\hline
\end{tabular}

${ }^{a}$ Kinematic; ${ }^{b}$ Herbig \& Jones (1983); ${ }^{c}$ assumed; ${ }^{d}$ Chernin \& Welch (1995).

edge of the distribution, too low for effective CS excitation. The densities in the regions of line formation derived from multitransitional data comprise usually $\sim 10^{6} \mathrm{~cm}^{-3}$ (e.g. Bergin et al. 1996; Plume et al. 1997). It is worth noting that the latter estimates are obtained assuming collisional excitation of CS molecules. One might think that in the vicinity of powerful IR sources radiative excitation via the lowest vibrational states can be important. However, as shown by Carroll \& Goldsmith (1981), such IR pumping can affect the CS excitation only in regions of $r \lesssim 0.04 \mathrm{pc}$ around an IR source. This is much less than the typical size of the CS cores. Then, one can see in Fig. 2 that in many cases the CS emission peaks do not coincide with the IRAS sources; the displacements exceed the uncertainties in the IRAS positions which are typically $\sim 10^{\prime \prime}-20^{\prime \prime}$. Thus, the IR pumping is probably not important for most of our cores. Apparently, the significant difference between the mean densities and densities in regions of line formation implies strong density inhomogeneities in the cores. The fact that the mean densities are frequently lower than densities needed for noticeable CS excitation indicates that there must be practically empty 

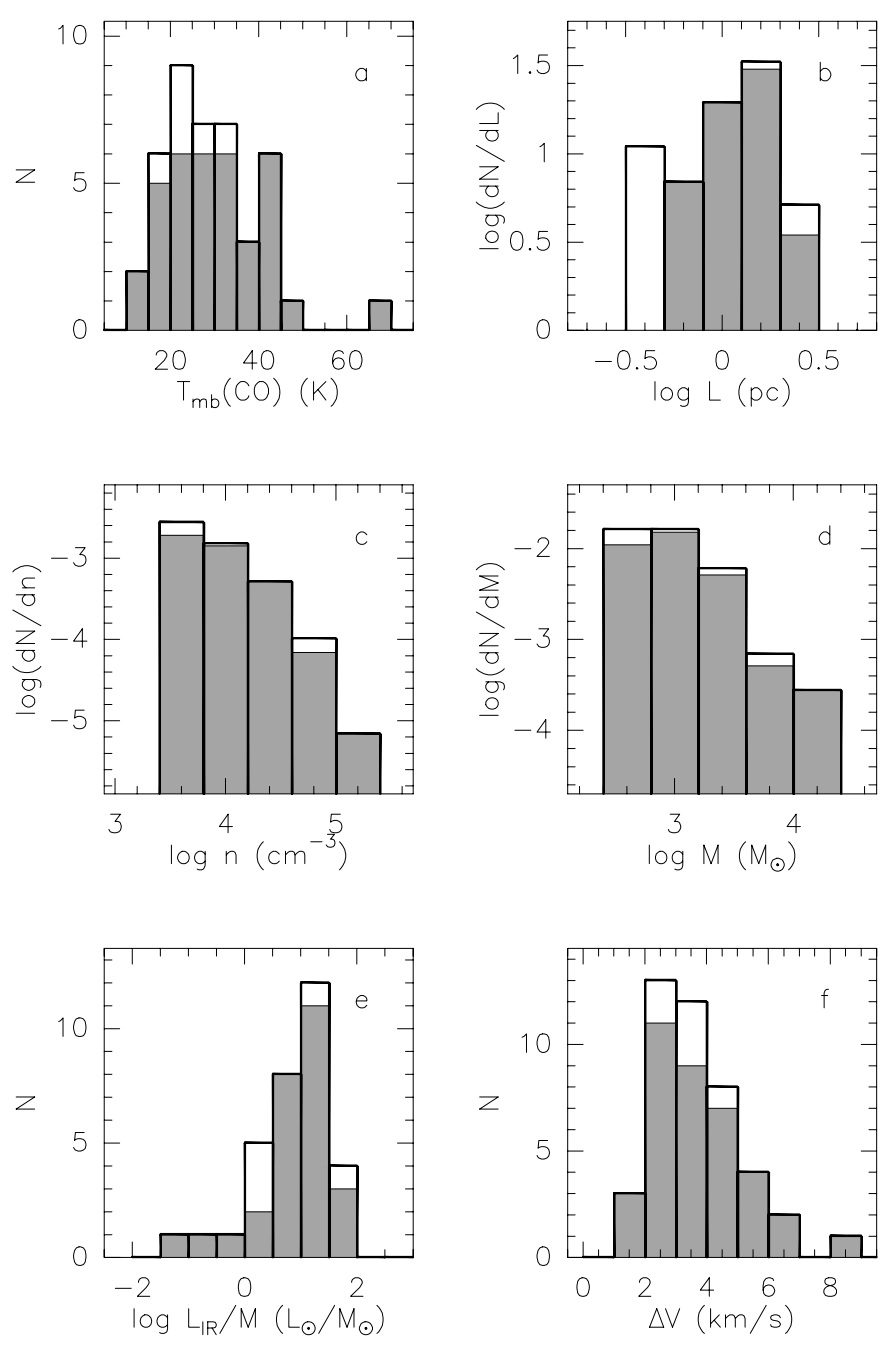

Fig. 3. Histograms of the peak $\mathrm{CO}$ main beam brightness temperature a), size b), mean density c), mass d), IR luminosity to mass ratio e) and mean CS line width $\mathbf{f}$ ) distributions for the CS cores observed at SEST and in Onsala located in the range $1-4 \mathrm{kpc}$ from the sun (filled) and within $5 \mathrm{kpc}$ from the sun (thick lines)

voids in the cores and it is easy to see that $1-2$ orders of magnitude difference between the mean density and the density in the emitting regions implies a correspondingly low volume filling factor for the CS emitting clumps.

The mass spectrum $\mathrm{d} N / \mathrm{d} M$ for $M \gtrsim 1000 M_{\odot}$ can be approximated by a power law $\mathrm{d} N / \mathrm{d} M \propto M^{-\alpha}$ with $\alpha=1.6 \pm 0.3$. This is very close to the slopes of clump mass spectra of individual molecular clouds which have been subject of several investigations (see, e.g., Blitz 1991 and references therein). They gave $\alpha$ from 1.4 to 1.7 for several giant molecular clouds. Most recently Kramer et al. (1998) found for 7 clouds that the $\alpha$ values lie between 1.6 and 1.8. However, our result does not refer to clumps in an individual cloud but to a sample of objects spread throughout the Galaxy. Moreover, the ranges of masses investigated in these studies are very different, $\sim 10^{-4}$
$10^{0} M_{\odot}$ in L 1457 (Kramer et al. 1998) as compared to $\sim 10^{3}-310^{4} M_{\odot}$ in the present work. The similarity in the slopes of the mass spectra in all these cases shows that it is really a universal law (unfortunately, unexplained yet).

The IR luminosity to mass ratio peaks at $\sim 10 L_{\odot} / M_{\odot}$ (we use here only the IRAS data of sufficiently high quality; the data with upper limits at 60 or $100 \mu \mathrm{m}$ were omitted). Recently Plume et al. (1997) found an average value of $190 L_{\odot} / M_{\odot}$ for objects of the same class which is an order of magnitude higher. The difference can probably be explained by the following factors:

(1) Plume et al. derive sizes and masses from the CS $J=5-4$ data. They are usually lower than the corresponding parameters derived from the $J=2-1$ line (e.g. Lapinov et al. 1998) since in higher transitions they see a smaller portion of the cloud. Most probably this is explained by density gradients in the cores (higher transitions require higher densities for excitation).

(2) Plume's et al. sources for which they derive the $L_{\mathrm{IR}} / M$ ratio are located mainly in the inner Galaxy while our distribution refers both to the inner and outer parts of the Galaxy. We show below that there is a significant gradient in this ratio along the galactocentric radius.

(3) Most of the sources selected by Plume et al. for this analysis belong to the very luminous objects with high star formation activity.

The distribution of the CS line widths which reflects the velocity dispersion in the cores shows at first that the internal movements are highly supersonic. If we recall the conclusion of a very low volume filling factor for the emitting clumps it would mean most probably that the line widths correspond to relative motions of these clumps. There are many arguments in favour of this model. Their discussion is beyond the scope of the present paper. Some of them concern the line broadening which is considered below.

\subsection{Galactic gradients of the core parameters}

In Paper II we found a trend for decreasing mean density of the cores with increasing galactocentric distance $R$. The data were limited to a rather narrow interval, $R \approx 7-11 \mathrm{kpc}$. The present Onsala results extend this interval significantly. In addition, Juvela (1996) performed similar observations in the inner Galaxy $(R \approx 4-8 \mathrm{kpc})$. However, he used additional selection criteria for the IR flux so that we cannot directly incorporate his data. Some of the Onsala sources lie as far as at $R \sim 20 \mathrm{kpc}$. However, we exclude these very distant objects and limit ourselves to $R \lesssim 15 \mathrm{kpc}$.

The distribution of the investigated objects in the galactic plane is shown in Fig. 4 where we include also Juvela's sources.

In Fig. 5 we plot the core parameters (peak CO main beam temperature, size, density, mass and mean CS line 


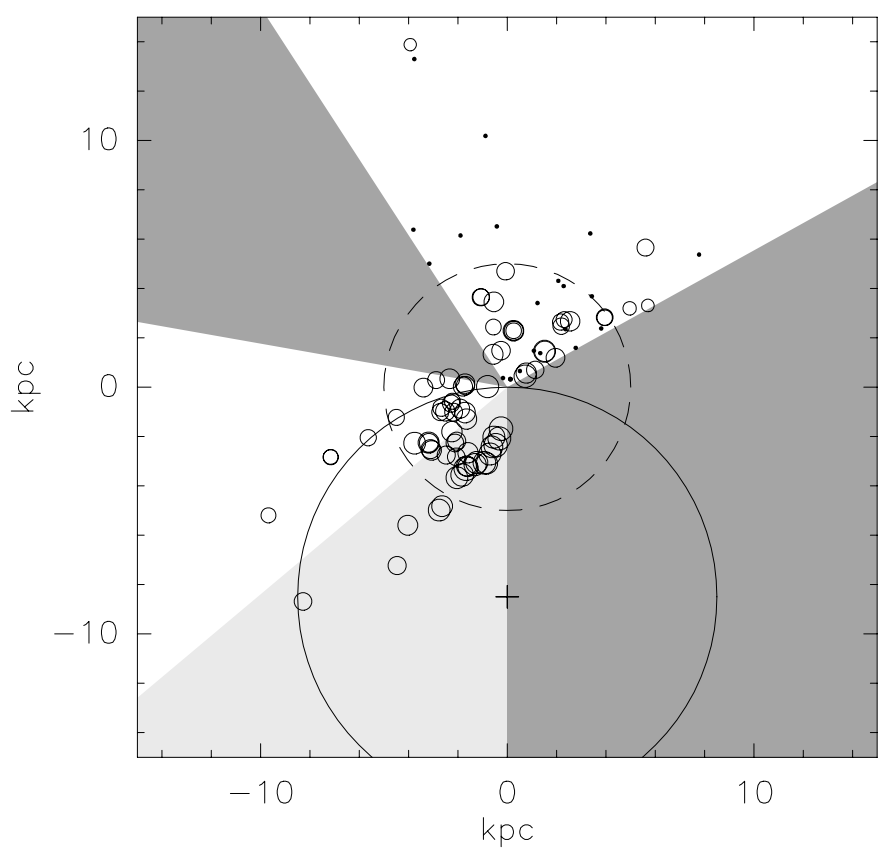

Fig. 4. Distribution of the detected sources in the galactic plane. The lightly shaded area corresponds to the range of galactic longitudes investigated by Juvela (1996). The heavily shaded part has not been included in our study. The size of the markers is proportional to $\log \bar{n}$ for the cases where the density has been derived. In other cases the objects are shown by dots. The solid line shows the solar circle. The dashed circle corresponds to the distance $5 \mathrm{kpc}$ from the sun

width) in dependence on the galactocentric radius using the SEST, Onsala and Metsähovi data. We use here the category II data for mass and density (i.e. assuming LTE mass equal to virial mass) since as shown above the assumption of $M=M_{\text {vir }}$ is in general correct. An inspection of Fig. 5 shows that these data (marked by dots) are well aligned with the other results and do not influence significantly the dependences. The Metsähovi estimates of the mass and density have been slightly corrected using the same procedure as for the other data. Then, for NGC 281 we use our new $\mathrm{C}^{34} \mathrm{~S}$ results (Table 3 ).

The most noticeable feature in the plots is the apparent decrease of the mean density with $R$. It seems to be linear in the $\log (\bar{n})-R$ coordinates so we approximated the dependence by the function

$\bar{n}=n_{0} \mathrm{e}^{-\left(R / R_{\mathrm{n}}\right)}$.

The best least squares estimates for the parameters are $n_{0}=(3.7 \pm 0.5) 10^{5} \mathrm{~cm}^{-3}, R_{n}=2.7 \pm 0.6 \mathrm{kpc}$. If we consider only the sources within $5 \mathrm{kpc}$ from the sun we obtain $n_{0}=(2.2 \pm 0.3) 10^{5} \mathrm{~cm}^{-3}, R_{n}=3.4 \pm 0.8 \mathrm{kpc}$. The Pearson's correlation coefficient in both cases is $r \approx-0.5$. Using the standard statistical methods (e.g., Bendat \& Piersol 1986) we obtain that the hypothesis of zero correlation is rejected at $\sim 0.002 \%$ level of significance for the whole sample, i.e. the correlation is very significant.

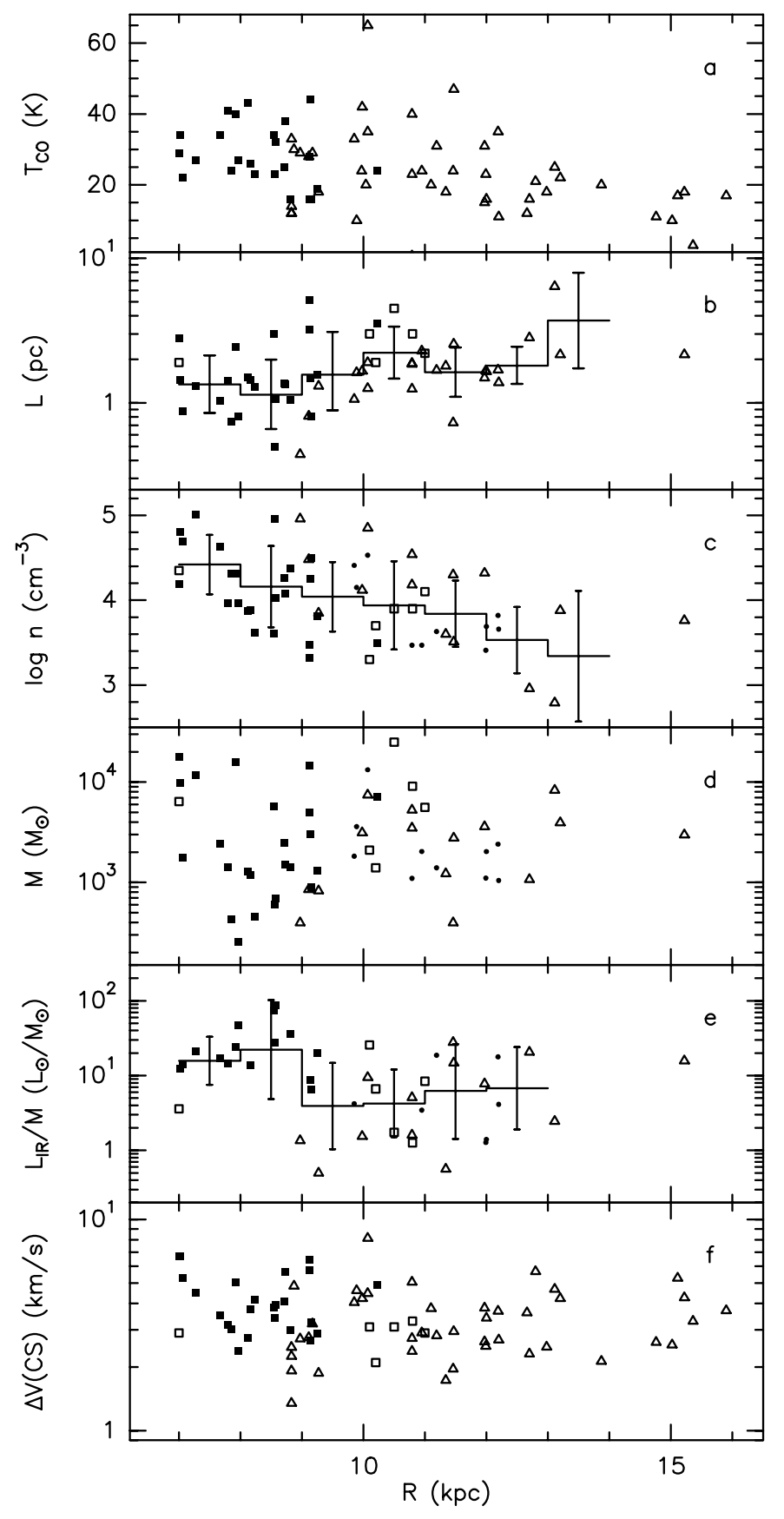

Fig. 5. Dependences of the peak CO main beam brightness temperature a), size b), mean density c), mass d), IR luminosity to mass ratio e) and mean CS line width f) on the galactocentric distances for the CS cores observed at SEST (filled squares), Onsala (triangles and dots; dots mark the category II data for mass and density, the other data are indicated by triangles) and Metsähovi radio telescope (open squares). The broken lines in panels b), c), e) show the average values in $1 \mathrm{kpc}$ bins. The error bars correspond to standard deviations of the data in the bins 


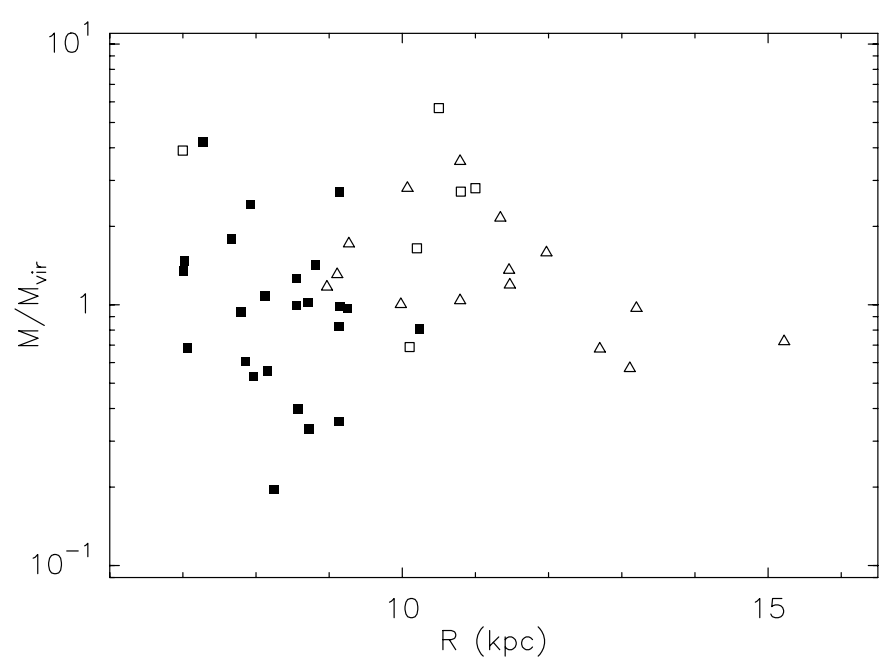

Fig. 6. The ratio of LTE mass to virial mass versus the galactocentric distance. The marks are the same as in Fig. 5

However, strictly speaking this conclusion is valid assuming binormal distribution of the variables. In our case the distributions differ seemingly from that. Bearing this in mind we consider also nonparametric or rank correlation which is more robust than linear correlation (Numerical Recipes 1992). For the $R-\log \bar{n}$ relationship the result is practically the same: the Spearman rankorder correlation coefficient is $r_{\mathrm{s}} \approx-0.50$ and the hypothesis of zero correlation is rejected at $\sim 0.01 \%$ level of significance.

As discussed in Paper II this trend should not be due to the beam dilution or selection effects because their influence should be more or less symmetric relative to the Sun position. In principle, such a trend could arise from a possible galactic gradient of CS abundance since the LTE masses are calculated assuming a constant value of $\chi(\mathrm{CS})=410^{-9}$ (Irvine et al. 1987). However, in this case we would see a similar trend in the $M / M_{\text {vir }}$ ratio because $M$ depends on $\chi(\mathrm{CS})$ while $M_{\text {vir }}$ does not. We found no dependence of this ratio on $R$ as can be seen in Fig. 6, which excludes a significant gradient in the CS abundance.

There is no dependence of the core mass on $R$. At the same time there is a noticeable increase of the core size with $R$ which is consistent with the density decrease and constant mass. The Spearman correlation coefficient is $r_{\mathrm{s}} \approx 0.37$ and the hypothesis of zero correlation is rejected at $\sim 0.5 \%$ level of significance. Another parameter which changes noticeably is the $L_{\mathrm{IR}} / M$ ratio. In this case $r_{\mathrm{s}} \approx$ -0.35 but the number of the data points is lower than in the previous cases. The hypothesis of zero correlation is rejected at $\sim 3 \%$ level of significance. This ratio varies in about the same proportion as the mean density. The leastsquares fit gives the scale length $R_{\mathrm{L} / \mathrm{M}}=3.6 \pm 1.2 \mathrm{kpc}$. It is worth noting the the robust linear fit to the data minimizing absolute deviations (Numerical Recipes 1992) gives the scale length of about $2.9 \mathrm{kpc}$ for both $R-\bar{n}$ and $R-L_{\mathrm{IR}} / M$ dependences.

The CO brightness temperature and CS line width do not change significantly in the considered range of galactocentric distances ( $7 \mathrm{kpc} \lesssim R \lesssim 15 \mathrm{kpc}$ ). As mentioned above, Juvela (1996) performed similar observations in the inner Galaxy. Since he selected the most IR luminous objects we cannot incorporate his data directly. Anyway, the comparison of his results with our data is rather informative and we present this in Fig. 7. Here we limit ourselves to the sources located within $5 \mathrm{kpc}$ from the sun. Then, we do not use the original Juvela's density estimates but the densities derived from virial masses as for our category II sources. The masses are also represented by virial masses. We prefer to do it in this way since Juvela estimated masses and densities from $\mathrm{C}^{34} \mathrm{~S}$ maps, not from CS maps as in our case. For the same reason we increased Juvela's estimates of sizes by a factor of 1.3 which is a mean ratio of the CS sizes to $\mathrm{C}^{34} \mathrm{~S}$ ones.

One can see that Juvela's points are consistent with the trends mentioned above, i.e. the gradients in the mean density and $L_{\mathrm{IR}} / M$ ratio. In addition, in Juvela's sample the line widths are noticeably larger on the average. There are practically no sources with such broad lines among those studied in Paper I and here. However, due the additional selection by IR flux we cannot exclude the possibility that Juvela's points represent only the upper parts of the distributions.

To summarize, the available data are consistent with an exponential decrease of the mean core density with the galactocentric radius for $R \approx 7-14 \mathrm{kpc}$. The characteristic scale length is $\sim 3 \mathrm{kpc}$. It is accompanied by a corresponding increase of the core size. The IR luminosity to mass ratio changes probably in about the same proportion as the mean density. The velocity dispersion in the cores probably increases towards the inner Galaxy at least at $R \lesssim 7 \mathrm{kpc}$.

How does this relate to other studies? Several years ago we found a strong gradient in the $\mathrm{HCN}$ detection rate versus the galactocentric radius for a sample of molecular clouds associated with Sharpless H II regions (Zinchenko et al. 1989). It is probably consistent with the density gradient found here (a more definite conclusion should rely on HCN excitation analysis).

Galactic gradients of molecular cloud properties have been a subject of several other studies in recent years (see, e.g., discussions in Helfer \& Blitz 1997 and Sakamoto et al. 1997). Briefly, the results are somewhat contradictory but there are unambiguous gradients in the $\mathrm{HCN} / \mathrm{CO}, \mathrm{CS} / \mathrm{CO}$ (Helfer \& Blitz 1997) and $\mathrm{HCO}^{+} /{ }^{13} \mathrm{CO}$ (Liszt 1995) emission line ratios which resemble our $\mathrm{HCN}$ result mentioned above. While Liszt interprets his result as an abundance effect, Helfer \& Blitz conclude that the contrast between the bulge and the disk is most likely caused by a 


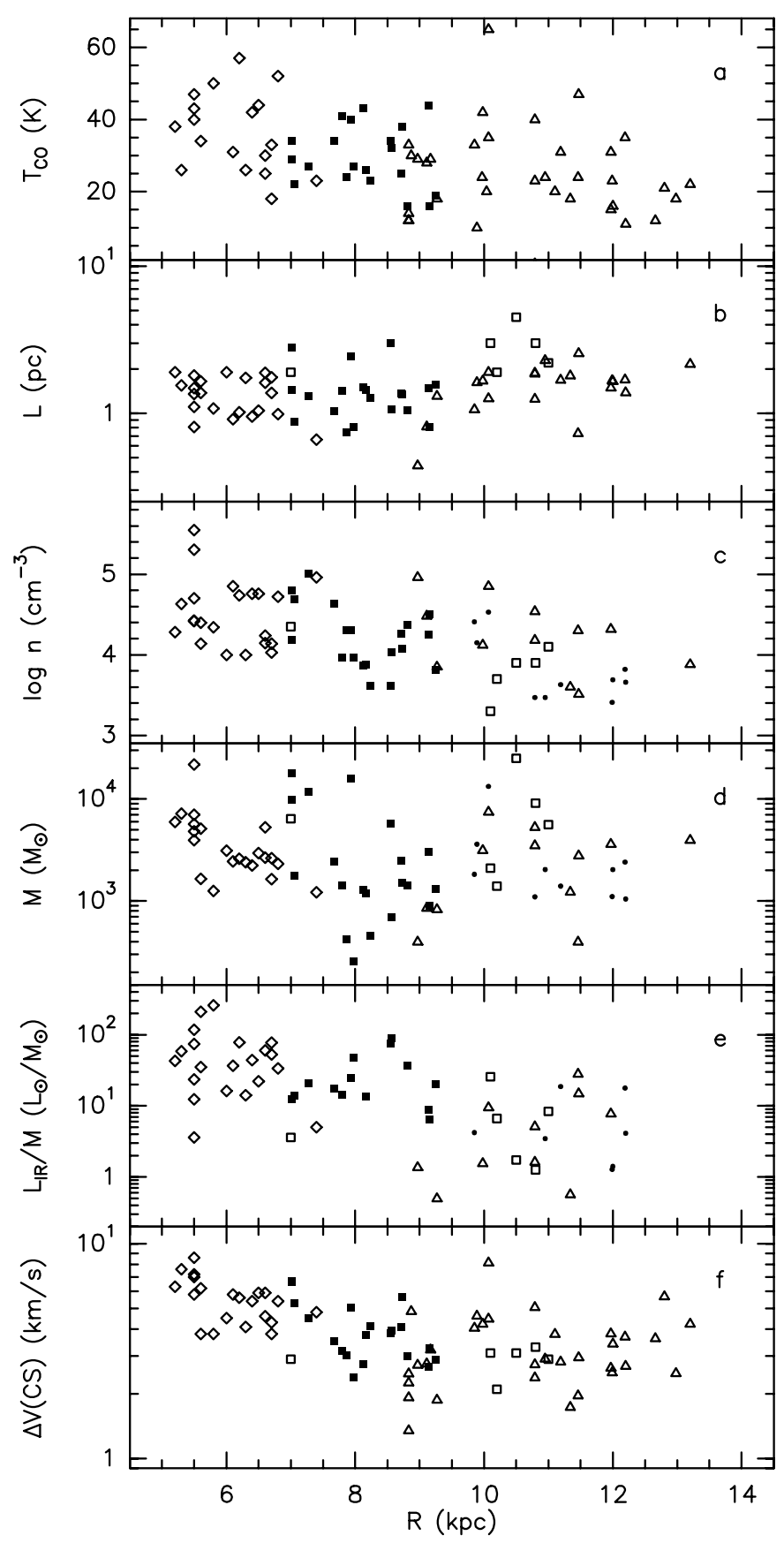

Fig. 7. The same as in Fig. 5 with inclusion of Juvela's (1996) data (diamonds) and with a distance limit of $d<5 \mathrm{kpc}$

combination of higher gas densities as well as higher kinetic temperatures in the bulge.

The most recent extensive CO $J=2-1$ survey by Sakamoto et al. (1997), when combined with the Columbia CO $J=1-0$ survey performed with the same angular resolution, shows a gradient in the $\mathrm{CO}(J=2-1) / \mathrm{CO}(J=$ $1-0)$ line ratio which is interpreted by the authors as an evidence of gradient in the high-density to low-density gas ratio.
Kislyakov \& Turner (1995) found no gradient in the ratio of the $\mathrm{C}^{18} \mathrm{O}(J=2-1)$ and $\mathrm{C}^{18} \mathrm{O}(J=1-0)$ line intensities for a sample of 11 clouds associated with Sharpless $\mathrm{H}$ II regions. Even if correct despite the low volume of the sample, this result does not contradict our findings since this ratio is governed by the gas density in regions of line formation while our result refers to the mean gas density in the source. If the objects are clumpy the mean density can change while the $\mathrm{C}^{18} \mathrm{O}(J=2-1) / \mathrm{C}^{18} \mathrm{O}(J=1-0)$ line ratio remains constant.

There are other indications for a galactic gradient in the cloud properties. E.g. a recent survey of thermal $\mathrm{SiO}$ emission (indicative of shocks) by Harju et al. (1998) shows much higher $\mathrm{SiO}$ line intensities in the inner Galaxy.

It is also worth noting that the exponential component of the stellar galactic disk has a scale length of $\sim 2.6 \mathrm{kpc}$ (Freudenreich 1998) which is very close to our value for the mean density distribution. At last, we note that the overall distribution of molecular gas traced by $\mathrm{CO}$ can be more appropriately described as a truncated exponential with a scale length of $2.8 \mathrm{kpc}$ than a "molecular ring" (Blitz 1997). Thus, we obtain a picture where both the total surface density of molecular gas as well as the mean density of the cores drop with increasing galactocentric radius according to an exponential law with a scale length of $\sim 3 \mathrm{kpc}$. The star formation efficiency, as described by the IR luminosity to mass ratio, behaves seemingly in a similar way. Most probably these coincidences are not accidental.

What can be the physical reason for this behaviour? We suppose that the most probable explanation involves an influence of galactic density waves responsible for the formation of the spiral structure as suggested already by Sakamoto et al. (1997). The normal velocity at which gas enters the shock front associated with the density wave is higher in the inner Galaxy and also the frequency at which the shock waves of an $m$-armed spiral encounter the gas increases inwards leading to higher degree of gas compression.

\subsection{Comparison of the $C S$ and $C^{34} S$ data}

We can obtain important implicit information about the internal core structure by comparing intensities and line widths of different isotopomers (e.g. Zinchenko et al. 1994). In general the line widths are closer to each other in clumpy clouds. In Fig. 8 the ratio $\Delta V(\mathrm{CS}) / \Delta V\left(\mathrm{C}^{34} \mathrm{~S}\right)$ is plotted versus the $T_{\mathrm{mb}}\left(\mathrm{C}^{34} \mathrm{~S}\right) / T_{\mathrm{mb}}(\mathrm{CS})$ ratio. We use here only the SEST and Onsala data with the highest signal to noise ratios and apparently simple line profiles. The solid line corresponds to an uniform LTE model with the terrestrial abundance ratio (22.5). The line broadening was calculated as in Zinchenko et al. (1994). The dashed line corresponds to the ratio of the effective optical depths in $\mathrm{CS}$ and $\mathrm{C}^{34} \mathrm{~S}$ lines equal to 10 . The reduction of this ratio 


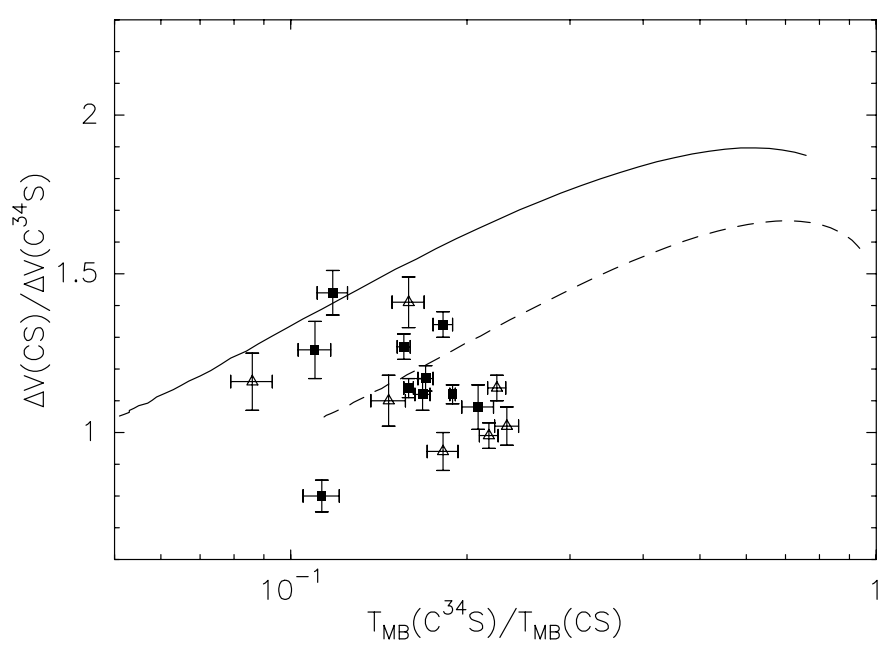

Fig. 8. The ratios of the $\mathrm{CS}$ and $\mathrm{C}^{34} \mathrm{~S}$ line widths versus $T_{\mathrm{mb}}\left(\mathrm{C}^{34} \mathrm{~S}\right) / T_{\mathrm{mb}}(\mathrm{CS})$ ratio for the SEST (filled squares) and Onsala (triangles) samples. The solid line corresponds to an uniform LTE model with the terrestrial abundance ratio. The dashed line corresponds to a model with the ratio of the effective optical depths in the two lines equal to 10

in respect to the terrestrial value can be caused either by the abundance effects or by clumpiness (e.g. Martin et al. 1984).

Though a few points lie close to the predictions of the first model and even more are closer to the second one, in general there is no systematic trend for increasing the line width ratio with increasing $T_{\mathrm{mb}}\left(\mathrm{C}^{34} \mathrm{~S}\right) / T_{\mathrm{mb}}(\mathrm{CS})$ ratio. So we can apparently reject an uniform model with any ratio of the optical depths and concentrate on other alternatives. In the framework of clumpy models we need apparently a set of clumps with different ratios of the optical depths. It seems to be possible to reproduce the presented results by varying the clump properties and the number of clumps on the line of sight. However, the discussion of clumpy models is beyond the scope of this paper. One of the main caveats on this way is the apparent smoothness of the line profiles as discussed e.g. recently by Tauber (1996). We leave a quantitative analysis until further publications.

Attempts for alternative explanations can be based on the assumption of line broadening by systematic motions or on non-uniform models with a smooth density distribution. The main kinds of systematic motions include collapse, expansion, rotation and outflows. The line widths of different isotopomers will be naturally close to each other in this case. The usual statistical arguments against the collapse as a main line broadening factor can be overwhelmed perhaps if we take into account that most of the contracting mass is ejected again via high-velocity outflows which are common in these regions (as suggested by A.V. Lapinov in a private communication). However, collapse should produce "kinematic signs" on the line profiles, e.g. red-shifted (or in some cases blue-shifted as shown by

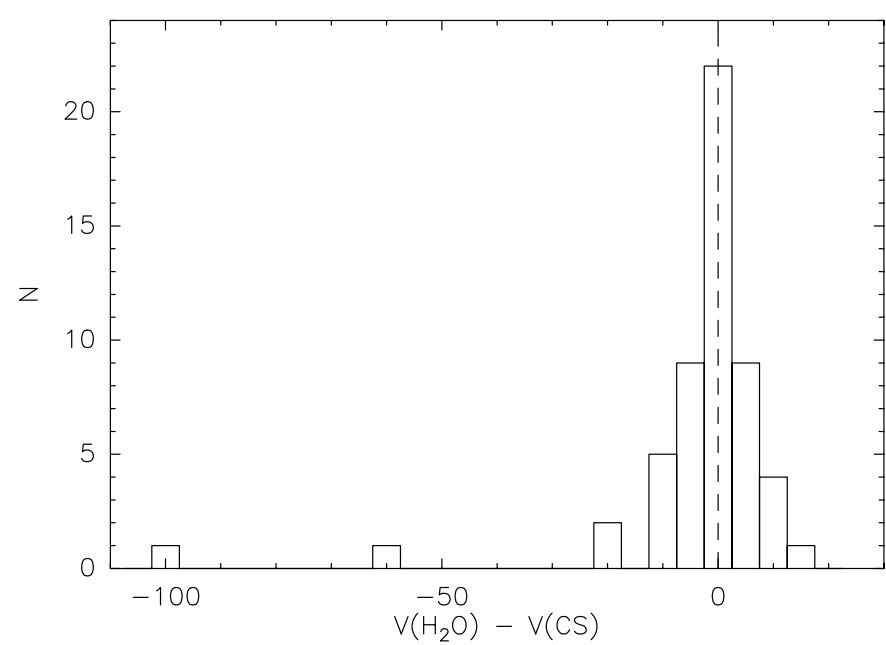

Fig. 9. Histogram of the differences between the $\mathrm{H}_{2} \mathrm{O}$ and $\mathrm{CS}$ velocities for the combination of the SEST and Onsala samples

Zinchenko \& Lapinov 1985) self-absorption dips which are not evident in our sample.

In microturbulent non-uniform models one can assume that the core itself is optically thin in both lines but the CS line is weakened in a diffuse envelope leading to an increase of the $T_{\mathrm{mb}}\left(\mathrm{C}^{34} \mathrm{~S}\right) / T_{\mathrm{mb}}(\mathrm{CS})$ ratio. It seems to be necessary to assume that the velocity dispersion in the envelope is much higher than in the core which is unrealistic. Furthermore, the measured $\mathrm{C}^{34} \mathrm{~S}$ column densities imply high optical depths in the CS lines.

We conclude that small scale clumpiness seems to be the most plausible explanation for the presented results with systematic motions being the best possible alternative.

\section{6. $\mathrm{CS}$ cores and $\mathrm{H}_{2} \mathrm{O}$ masers}

Our SEST data as well as Juvela's data have shown a slight asymmetry in the distribution of the velocity difference of the masers and CS cores with the maser velocities being more negative on the average. In the present Onsala sample we do not see such asymmetry. In Fig. 9 we plot the histogram of $V\left(\mathrm{H}_{2} \mathrm{O}\right)-V(\mathrm{CS})$ values for the combined SEST+Onsala data set. The distribution is practically symmetric. However, this plot shows that the difference in the velocities can be quite large (the standard deviation is $\sim 7 \mathrm{~km} \mathrm{~s}^{-1}$ if we neglect 2 masers with the highest velocity differences). Thus, $\mathrm{H}_{2} \mathrm{O}$ masers are moving with high velocities relative the CS cores.

\section{Summary and conclusions}

We have presented results of the CS $J=2-1$ survey towards 55 northern non-stellar $\mathrm{H}_{2} \mathrm{O}$ masers. We detected 47 CS cores associated probably with 50 masers. The CS emission for this sample is weaker on the average than for 
the similar southern sample studied by us earlier at SEST. CS maps as well as CO, CS and when available $\mathrm{C}^{34} \mathrm{~S}$ spectra have been presented for 26 best studied cores.

From the CS maps and optically thin $\mathrm{C}^{34} \mathrm{~S}$ emission we have derived the basic physical parameters of the cores: size, LTE mass, mean density, virial mass. Combining the present results with the previous SEST data we obtained statistical distributions of the core parameters. The CO brightness temperature for most cores lies in the range $\sim 15-50 \mathrm{~K}$ with a peak at $20-30 \mathrm{~K}$. The size of most cores is $L \sim 1.0-1.5 \mathrm{pc}$. The mean density lies in the range $n \sim 10^{3}-10^{5} \mathrm{~cm}^{-3}$ which is much lower than densities needed for CS excitation from multitransitional analysis. The slope of the mass spectrum for $M \gtrsim 1000 M_{\odot}$ is $1.6 \pm 0.3$. The ratio of the IR luminosity of associated IRAS point sources to mass peaks at $\sim 10 L_{\odot} / M_{\odot}$. The CS line widths are highly supersonic $\left(\sim 1.5-9 \mathrm{~km} \mathrm{~s}^{-1}\right)$.

We have analysed the dependences of these parameters on galactocentric distance $R$. The mean density of the cores decreases with increasing $R$ in agreement with an exponential law with a scale length of $\sim 3 \mathrm{kpc}$ in the interval $R \approx 7-14 \mathrm{kpc}$. The IR luminosity to mass ratio changes probably in a similar way. The core size increases with $R$ in accordance with the density decrease and constant mass.

The comparison of the $\mathrm{CS}$ and $\mathrm{C}^{34} \mathrm{~S}$ data shows almost no broadening of the CS lines due to optical depth effects. This can be explained probably by small scale clumpiness in the cores. The velocity difference between the CS cores and $\mathrm{H}_{2} \mathrm{O}$ masers is close to zero on the average with the standard deviation of $\sim 7 \mathrm{~km} \mathrm{~s}^{-1}$.

Acknowledgements. We are very grateful to Dr. Lars E.B. Johansson for his help with the observations and for obtaining important additional data, to Prof. Kalevi Mattila for critical reading of the manuscript and to an anonymous referee for valuable suggestions. I.Z. and L.P. thank the Helsinki University Observatory for the hospitality during the data reduction and the paper preparation stages. They were also supported in part by the INTAS grant 93-2168-ext and grant 9602-16472 from the Russian Foundation for Basic Research. This research has made use of the Simbad database, operated at CDS, Strasbourg, France.

\section{References}

Anglada G., Estalella R., Pastor J., 1996, ApJ 463, 205

Bendat J.S., Piersol A.G., 1986, Random Data. Analysis and Measurement Procedures. Wiley, New York

Bergin E.A., Snell R.L., Goldsmith P.F., 1996, ApJ 460, 343
Blitz L., 1991, Star forming giant molecular clouds. In: Lada C.J., Kylafis N.D. (eds.) The Physics of Star Formation and Early Stellar Evolution. Kluwer Acad. Publ., Dordrecht

Blitz L., 1997, CO in the Milky Way. In: Latter W.B., Radford S.J.E., Jewell P.R., Mangum J.G., Bally J. (eds.) CO: Twenty-Five Years of Millimeter-Wave Spectroscopy. Kluwer Acad. Publ., Dordrecht

Brand J., Blitz L., 1993, A\&A 275, 67

Brand J., Cesaroni R., Caselli P., et al., 1994, A\&AS 103, 541

Bronfman L., Nyman L.-Å., May J., 1996, A\&AS 115, 81

Carroll T.J., Goldsmith P.F., 1981, ApJ 245, 891

Chernin L.M., Welch W.J., 1995, ApJ 440, L21

Comoretto G., Palagi F., Cesaroni R., et al., 1990, A\&AS 84, 179

Fich M., Blitz L., Stark A.A., 1989, ApJ 342, 272

Freudenreich H.T., 1998, ApJ 492, 495

Harju J., Lehtinen K., Booth R., Zinchenko I., 1998, A\&AS (in press)

Helfer T.T., Blitz L., 1997, ApJ 478, 233

Herbig G.H., Jones B.F., 1983, AJ 88, 1040

Irvine W.M., Goldsmith P.F., Hjalmarson A., 1987, Chemical Abundances in Molecular Clouds. In: Hollenbach D.L., Thronson Jr. H.A. (eds.) Interstellar Processes. Reidel, Dordrecht

Juvela M., 1996, A\&AS 118, 191

Kislyakov A.G., Turner B.E., 1995, AZh 72, 168

Kramer C., Stutzki J., Röhrig R., Corneliussen U., 1998, A\&A 329,249

Lapinov A.V., Schilke P., Juvela M., Zinchenko I., 1998, A\&A 336, 1007

Liszt H.S., 1995, ApJ 442, 163

Martin H.M., Sanders D.B., Hills R.E., 1984, MNRAS 208, 35

Numerical Recipes in Fortran, 1992, Press W.H., Teukolsky S.A., Vetterling W.T., Flannery B.P. (eds.). Cambridge Univ. Press

Palagi F., Cesaroni R., Comoretto G., Felli M., Natale V., 1993, A\&AS 101, 153

Plume R., Jaffe D.T., Evans II N.J., 1992, ApJS 78, 505

Plume R., Jaffe D.T., Evans II N.J., Martín-Pintado J., Gómez-González J., 1997, ApJ 476, 730

Sakamoto S., Hasegawa T., Handa T., Hayashi M., Oka T., 1997, ApJ 486, 276

Tauber J.A., 1996, A\&A 315, 591

Yang L., Umemoto T., Iwata T., Fukui Y., 1991, ApJ 373, 137

Zinchenko I., 1995, A\&A 303, 554 (Paper II)

Zinchenko I.I., Lapinov A.V., 1985, AZh 62, 860 (SvA 29, 502)

Zinchenko I.I., Lapinov A.V., Pirogov L.E., 1989, AZh 66, 1142 (SvA 33, 590)

Zinchenko I., Forsström V., Lapinov A., Mattila K., 1994, A\&A 288,601

Zinchenko I., Mattila K., Toriseva M., 1995, A\&AS 111, 95 (Paper I)

Zinchenko I., Henning Th., Schreyer K., 1997, A\&AS 124, 385 